\title{
Les variations des glaciers suisses en 1974-1975 et quelques indications sur les résultats récoltés pendant la Décennie Hydrologique Internationale de 1964-65 à 1973-74
}

PAR

P. Kasser

ET

M. Aellen

Laborntoires de Recherches hydrauliques. hydrologiques et glaciologiques de l'École l'olytechnique Fédérale à Zurich

\section{Introduction}

Les raisons suivantes ont incité à une analyse préliminaire des variations en $1974 / 75$ des glaciers des Alpes suisses :

- Pour la première fois depuis $1925 / 26$, plus de la moitié des glaciers observés est en crue.

- Le fait qu'en 1974/75 on dispose en même temps d'un d'un nombre considérable de glaciers en crue et de glaciers en décrue devrait permettre d'étudier les relations entre le signe de la variation et quelques paramètres morphologiques.

- Les questions citées ci-dessous sont importantes à la fois pour la mise en valeur des ressources hydrauliques et pour des problèmes de sécurité.

Le nombre croissant de glaciers en crue signifie-t-il que la longue période de récession soit terminée? Les glaciers sont-ils actuellement plus ou moins en équilibre avec le climat ? Est-ce qu'une période de crue a déjà commencé ?

\section{Le réseau d'observations et les résul- tats des mensurations en 1974/75}

\subsection{Le réseau d'observations}

Le réseau d'observations fondé en 1880 par F.A. Forel et développé depuis 1893 sous la responsabilité de la Commission des glaciers de la Société Helvétique des Sciences Naturelles avec le concours des Services Forestiers est présenté à la figure 1 . Les noms des glaciers qui corres- pondent aux numéros d'identification de la figure 1 se trouvent à la colonne G1. du tableau 1. Durant les décennies, le réseau a subi de nombreuses modifications. Il comprend actuellement 115 glaciers dont on observe les variations annuelles de longueur. Pour quatre glaciers, les bilans annuels de masse sont également mesurés (v. tableau 2b). Pour l'ensemble des glaciers dans le bassin versant de la Massa, soit les glaciers d'Aletsch, les bilans sont déterminés par les bilans hydrologiques; pour les trois autres glaciers, on mesure les bilans spécifiques annuels au moyen de balises d'accumulation et de balises d'ablation plantées à la surface du glacier. Des vols photogrammétriques répétés permettent de constater pour un certain nombre de glaciers les variations de volume et de surface en fonction de l'altitude pour des périodes de plusieurs années. Citons enfin les observations spéciales faites à différents glaciers en.vue d'un danger éventuel.

2.2. Les variations de longueur et les bilans de $1974 / 75$

Le tableau 1 contient les valeurs des variations de longueur mesurées aux différents glaciers en 1973/74 et en 1974/75. Pour l'année 1974/75, la récapitulation du tableau $2 \mathrm{a}$ montre 56 glaciers en crue, 11 glaciers stationnaires et 40 glaciers en décrue. Le rapport des glaciers en crue et en décrue varie avec la région. Dans les bassins versants de la Reuss, de la Limmat et du Tessin presque tous les glaciers ont avancé, tandis que dans le bassin du Rhône, le nombre des glaciers en décrue a dépassé un peu celui des glaciers croissants. Il semble que ce comportement différent provienne surtout des propriétés morphologiques régionales des glaciers observés. 


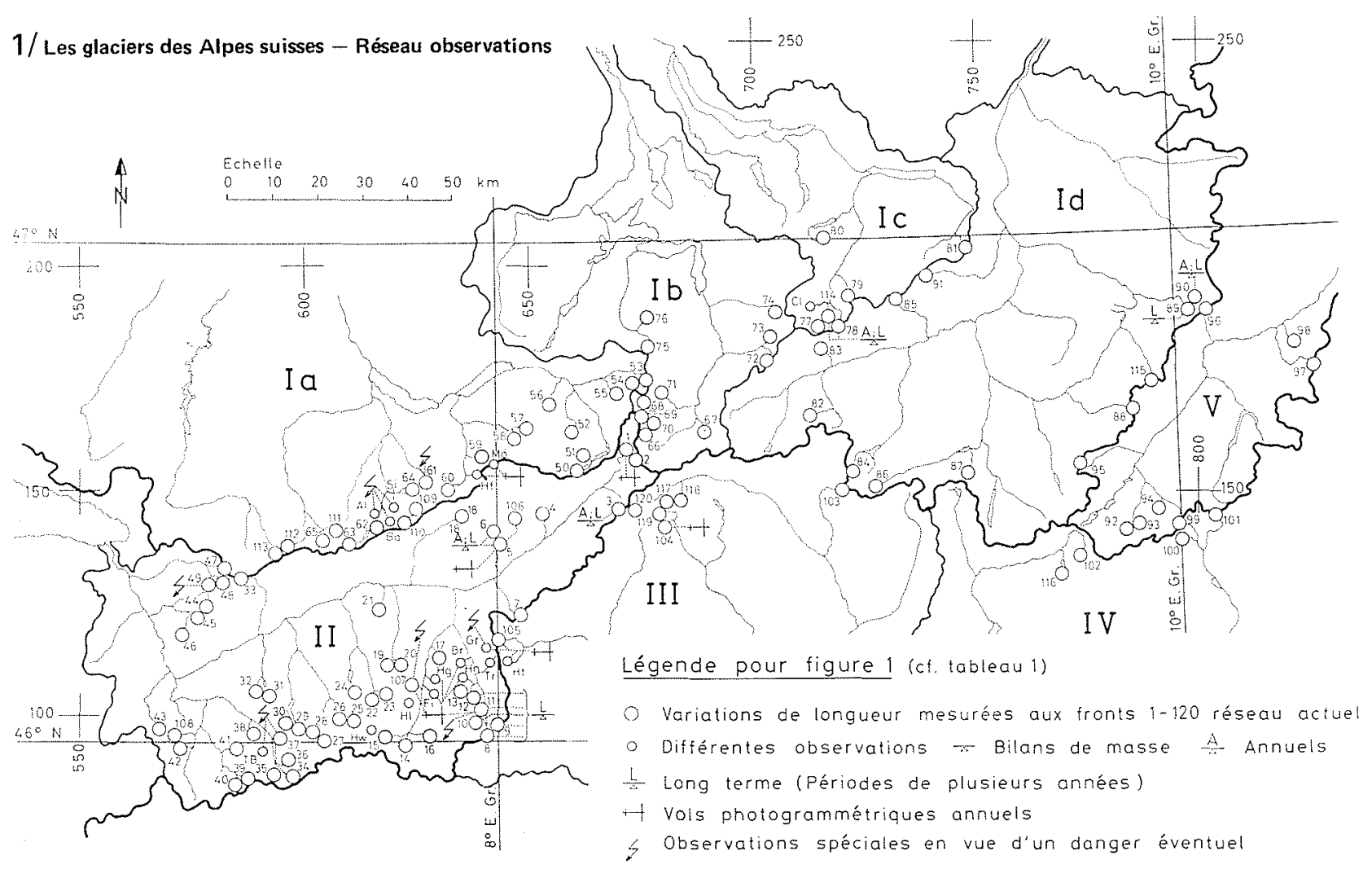

\begin{tabular}{|c|c|c|c|c|c|c|c|c|}
\hline \multicolumn{9}{|c|}{$\begin{array}{l}\text { Liste des glaciers du réseau d'observations avec quelques paramètres morphologíques et les } \\
\text { variations de longueur en } 1973 / 74 \text { et } 1974 / 75 \text { (v. fig. 1) }\end{array}$} \\
\hline \multicolumn{2}{|r|}{ no. } & \multirow{2}{*}{ glacier } & \multicolumn{4}{|c|}{ paramètres morphologiques } & \multicolumn{2}{|c|}{$\begin{array}{l}\text { variations de } \\
\text { longueur en }\end{array}$} \\
\hline Gl. & $\ln v$. & & $\begin{array}{c}\mathrm{S} \\
\mathrm{km}^{2}\end{array}$ & $\alpha$ & $\begin{array}{l}A_{f} \\
\text { m.m.m. }\end{array}$ & $\begin{array}{l}\text { expo- } \\
\text { sition }\end{array}$ & $\begin{array}{l}1973 / 74 \\
\mathrm{~m}\end{array}$ & $\begin{array}{c}1974 / 75 \\
\mathrm{~m}\end{array}$ \\
\hline (1) & $(2)$ & (3) & (4) & (5) & (6) & (7) & $(8)$ & (8) \\
\hline \multicolumn{9}{|c|}{ Bassin versant du Rhône } \\
\hline 1 & B $43 / 3$ & Rhône & 17.38 & 14.5 & 2140 & S & +2.8 & +17.0 \\
\hline 2 & B $44 / 3$ & Mutt & 0.57 & 38.2 & 2580 & NW & +2.1 & +3.4 \\
\hline 3 & B $45 / 4$ & Gries (Aegina) & 6.60 & 15.8 & 2380 & $\mathrm{NE}$ & -6.7 & -6.2 \\
\hline 4 & B $40 / 7$ & Fiescher & 33.06 & 16.0 & 1640 & $\mathrm{~S}$ & -23.8 & -30.1 \\
\hline 5 & B $36 / 26$ & Grosser Aletsch & 86.76 & 10.6 & 1520 & $\mathrm{~S}$ & -40.5 & -2.0 \\
\hline 106 & B $36 / 21$ & Mittelaletsch & 8.50 & 32.9 & 2260 & $\mathrm{SE}$ & -10.6 & -4.6 \\
\hline 6 & B $36 / 1$ & Oberaletsch & 21.71 & 18.5 & 2180 & $\mathrm{SE}$ & $-\quad 7.9$ & \\
\hline 7 & B $47 / 4$ & Kaltwasser & 1.85 & 46.2 & 2620 & $W$ & $\mathrm{n}$ & st \\
\hline 8 & B $52 / 20$ & Tälliboden & 0.26 & 37.5 & 2640 & NW & -0.2 & +8.6 \\
\hline 9 & B $52 / 17$ & Ofental & 0.40 & 40.0 & 2660 & $\mathrm{~N}$ & $\mathrm{n}$ & $\mathrm{n}$ \\
\hline 10 & B $52 / 24$ & Schwarzberg & 6.20 & 21.4 & 2660 & NE & -1.0 & +8.3 \\
\hline 11 & B $52 / 29$ & Allalin & 9.94 & 28.6 & 2340 & $\mathrm{E}$ & +10.8 & +44.0 \\
\hline 12 & B $52 / 33$ & Kessjen & 0.61 & 42.2 & 2840 & $N E$ & & $+4.7^{2 \mathrm{a}}$ \\
\hline 13 & B $53 / 4$ & Fee (Nord) & 16.66 & 45.5 & 2040 & NE & +17.0 & +15.0 \\
\hline 14 & B $56 / 7$ & Gorner & 68.86 & 17.6 & 2120 & NW & & $-62.6^{2 a}$ \\
\hline 15 & B $57 / 5$ & Zmutt & 16.98 & 22.5 & 2280 & $\mathrm{E}$ & -32.0 & -6.2 \\
\hline 16 & B $56 / 3$ & Findelen & 19.09 & 20.2 & 2320 & W & & $-34.7^{2 \mathrm{a}}$ \\
\hline 107 & B $58 / 8$ & $\mathrm{Bis}$ & 4.79 & 64.2 & 2060 & $E$ & $+x$ & $+x$ \\
\hline 17 & B $54 / 3$ & Ried & 8.26 & 35.2 & 2060 & NW & -9.1 & -4.5 \\
\hline 18 & B $31 / 4$ & Lang & 10.03 & 22.9 & 2140 & SW & & $-38.0^{2 a}$ \\
\hline 191 & \multirow{2}{*}{ B $60 / 9$} & Turtmann-West & 6.98 & 33.7 & 2260 & $\mathrm{~N}$ & +5.5 & +13.3 \\
\hline 201 & & Brunegg & 6.12 & 35.9 & 2440 & $N W$ & -1.6 & st \\
\hline 21 & B $61 / 2$ & Bella Tola & 0.31 & 50.0 & 2680 & $\mathrm{~N}$ & +0.6 & +18.3 \\
\hline 22 & B $63 / 5$ & Zinal & 16.24 & 26.8 & 2000 & $N$ & +33.0 & -82.0 \\
\hline 23 & B $62 / 10$ & Moming & 5.77 & 42.1 & 2460 & NW & $+x$ & -6.3 \\
\hline 24 & B $64 / 2$ & Moiry & 6.11 & 25.0 & 2420 & $\mathrm{~N}$ & & $-2.0^{2 \mathrm{a}}$ \\
\hline
\end{tabular}




\begin{tabular}{|c|c|c|c|c|c|c|c|c|}
\hline \multicolumn{2}{|c|}{ no. } & \multirow[t]{2}{*}{ glacier } & \multicolumn{4}{|c|}{ paramètres morphologiques } & \multicolumn{2}{|c|}{$\begin{array}{l}\text { variations de } \\
\text { longueur en }\end{array}$} \\
\hline Gl. & Inv. & & $\begin{array}{c}\mathrm{S} \\
\mathrm{km}^{2}\end{array}$ & $\alpha$ & $\begin{array}{c}A_{f} \\
\text { m.s.m. }\end{array}$ & $\begin{array}{l}\text { expo- } \\
\text { sition }\end{array}$ & $\begin{array}{c}1973 / 74 \\
\mathrm{~m}\end{array}$ & $\begin{array}{c}1974 / 75 \\
\mathrm{~m}\end{array}$ \\
\hline (1) & (2) & (3) & (4) & (5) & $(6)$ & (7) & (8) & $(8)$ \\
\hline \multicolumn{3}{|c|}{ Bassin versant du Rhône } & & & & & & \\
\hline 25 & B $72 / 11$ & Ferpècle & 9.79 & 25.3 & 2140 & $\mathrm{~N}$ & & $+1.8^{2 \mathrm{a}}$ \\
\hline 26 & В $72 / 15$ & Mont Miné & 10.89 & 21.5 & 1980 & $\mathrm{~N}$ & $+x$ & $+x$ \\
\hline 27 & B $73 / 14$ & Bas d'Arolla & 6.02 & 27.6 & 2140 & $\mathrm{~N}$ & +7.0 & +16.0 \\
\hline 28 & B $73 / 16$ & Tsidjiore Nouve & 3.12 & 30.0 & 2280 & NE & +8.0 & +13.0 \\
\hline 29 & B $74 / 8$ & Cheillon & 4.73 & 29.0 & 2660 & $\mathrm{~N}$ & & $-58.3^{2 \mathrm{a}}$ \\
\hline 30 & B $74 / 11$ & L'En Darrey & 1.86 & 47.5 & 2560 & $\mathrm{NE}$ & & $-52.7^{2 a}$ \\
\hline 31 & B $75 / 6$ & Grand Désert & 1.85 & 20.9 & 2760 & $\mathrm{~N}$ & & $-35.0^{2 \mathrm{a}}$ \\
\hline 32 & B $75 / 9$ & Mont Fort & 0.46 & 34.3 & 2800 & $\mathrm{NE}$ & & $-5.6^{2 \mathrm{a}}$ \\
\hline 33 & B $22 / 1$ & Tsanfleuron & 3.78 & 16.1 & 2440 & $\mathrm{E}$ & st. & -7.0 \\
\hline 34 & B $82 / 27$ & Otemma & 16.55 & 15.8 & 2460 & SW & & $-97.0^{2 \mathrm{a}}$ \\
\hline 35 & B $82 / 36$ & Mont Durand & 7.59 & 32.0 & 2280 & $\mathrm{E}$ & st. & -10.0 \\
\hline 36 & B $82 / 19$ & Breney & 9.80 & 19.7 & 2560 & SW & & $-33.0^{2 \mathrm{a}}$ \\
\hline 37 & B $82 / 14$ & Giétro & 5.94 & 23.7 & 2540 & NW & st. & +5.3 \\
\hline 38 & B $83 / 3$ & Corbassière & 17.44 & 21.2 & $22=0$ & $\mathrm{~N}$ & -12.0 & +9.0 \\
\hline 39 & B $84 / 15$ & Valsorey & 2.34 & 32.2 & 2400 & $\mathrm{~N}$ & & $+10.2^{2 \mathrm{a}}$ \\
\hline 40 & B $84 / 17$ & Tseudet & 1.73 & 40.7 & 2500 & $N$ & & $-22.0^{2 a}$ \\
\hline 41 & B $84 / 4$ & Boveyre & 1.99 & 40.0 & 2620 & NW & & $+11.0^{3 \mathrm{a}}$ \\
\hline 42 & B $85 / 16$ & Saleina & 5.03 & 33.1 & 1760 & $\mathrm{NE}$ & & $+33.5^{2 \mathrm{a}}$ \\
\hline 43 & B $90 / 2$ & Trient & 6.58 & 33.8 & 1800 & $\mathrm{~N}$ & +12.6 & +10.0 \\
\hline 44 & B $17 / 2$ & Paneyrosse & 0.45 & 51.4 & 2400 & $\mathrm{~N}$ & st. & $-x$ \\
\hline 45 & $\mathrm{~B} 17 / 3$ & Grand Plan Névé & 0.20 & 50.0 & 2360 & $\mathrm{~N}$ & st. & st. \\
\hline 46 & B $17 / 8$ & Martinets & 0.59 & 30.0 & 2140 & $\mathrm{NE}$ & st. & st. \\
\hline 47 & B $16 / 1$ & Sex Rouge & 0.72 & 25.0 & 2660 & NW & st. & $+2.8^{3 a}$ \\
\hline 48 & B $16 / 3$ & Prapio & 0.37 & 55.6 & 2500 & NW & & $-2.0^{3 a}$ \\
\hline 49 & B $16 / 5$ & Pierredar & 0.67 & 58.0 & 2380 & $\mathrm{~N}$ & +15.0 & $-x$ \\
\hline \multicolumn{3}{|c|}{ Bassin versant de l'Aar } & & & \\
\hline 50 & A $54 g / 3$ & Oberaar & 5.23 & 21.5 & 2300 & $\mathrm{E}$ & -24.0 & -6.9 \\
\hline 51 & A $54 g / 11$ & Unteraar & 28.41 & 16.1 & 1900 & $\mathrm{E}$ & -13.5 & -14.4 \\
\hline 52 & A $54 \mathrm{i} / 5$ & Gauli & 13.70 & 21.5 & 2140 & $\mathrm{E}$ & -21.5 & -8.8 \\
\hline 53 & A $54 \mathrm{e} / 12$ & Stein & 6.52 & 32.8 & 1960 & $\mathrm{~N}$ & & $+37.0^{2 a}$ \\
\hline 54 & A $54 \mathrm{e} / 13$ & Steinlimmi & 2.21 & 43.0 & 2140 & $\mathrm{~N}$ & & $+8.5^{2 a}$ \\
\hline 55 & A $54 \mathrm{e} / 24$ & Trift & 17.07 & 23.4 & 1720 & NW & -5.0 & st. \\
\hline 56 & A $54 \mathrm{j} / 2$ & Rosenlaui & 6.20 & 35.4 & 1860 & $\mathrm{NE}$ & -15.0 & $\mathrm{n}$ \\
\hline 57 & A $541 / 4$ & Oberer Grindelwald & 10.07 & 45.5 & 1240 & NW & +35.0 & +17.0 \\
\hline 58 & A $541 / 19$ & Unterer Grindelwald & 21.71 & 31.6 & 1260 & $N$ & $-x$ & $-x$ \\
\hline 59 & A $54 \mathrm{~m} / 3$ & Eiger & 2.27 & 73.1 & 2200 & NW & & $+24.9^{3 \mathrm{a}}$ \\
\hline 60 & A $54 \mathrm{~m} / 21$ & Tschingel & 6.18 & 33.2 & 2240 & $\mathrm{NE}$ & st. & +7.6 \\
\hline 61 & A $55 \mathrm{a} / 4$ & Gamchi & 1.73 & 31.9 & 1980 & $\mathrm{~N}$ & +4.3 & +8.6 \\
\hline 109 & A $55 \mathrm{~b} / 13$ & Alpetli & 14.02 & 13.8 & 2320 & SW & -0.7 & +2.3 \\
\hline 110 & A $55 b / 16$ & Lötschen & 0.91 & 28.0 & 2320 & $\mathrm{~N}$ & $-x$ & $\mathrm{n}$ \\
\hline 62 & A $55 c / 5$ & Schwarz & 1.60 & 36.9 & 2220 & NW & -1.7 & +0.8 \\
\hline 63 & A $55 c / 13$ & Lämmern & 3.35 & 28.0 & 2540 & $\mathrm{E}$ & -2.0 & +4.0 \\
\hline 64 & A $55 \mathrm{~b} / 2$ & Blümlisalp & 2.98 & 50.3 & 2200 & NW & +5.0 & $\mathrm{n}$ \\
\hline 111 & A $55 \mathrm{f} / 1$ & Ammerten & 1.89 & 31.4 & 2360 & NW & & $+7.5^{2 \mathrm{a}}$ \\
\hline 65 & A $55 \mathrm{f} / 3$ & Rätzli & 9.80 & 16.0 & 2320 & NW & & $-13.0^{2 \mathrm{a}}$ \\
\hline \multicolumn{3}{|c|}{ Bassin versant de la Reuss } & & & & & & \\
\hline 66 & A $51 \mathrm{e} / 37$ & Tiefen & 3.17 & 27.1 & 2480 & $S E$ & +3.7 & +3.0 \\
\hline 67 & A $51 \mathrm{e} / 12$ & St. Anna & 0.44 & 35.6 & 2580 & $N$ & -0.5 & +8.0 \\
\hline 68 & A $51 \mathrm{f} / 15$ & Chelen & 3.15 & 39.4 & 2120 & SE & +10.3 & +16.5 \\
\hline 69 & A $51 \mathrm{f} / 13$ & Rotfirn (Nord) & 1.21 & 59.1 & 2000 & $\mathrm{NE}$ & -6.0 & +4.5 \\
\hline 70 & A $51 \mathrm{f} / 10$ & Damma & 6.32 & 44.8 & 2040 & NE & +2.2 & +13.8 \\
\hline 71 & A $51 \mathrm{f} / 24$ & Wallenbur & 1.70 & 46.4 & 2260 & SE & -0.2 & $+x$ \\
\hline 72 & A $51 \mathrm{~d} / 15$ & Brunni & 2.99 & 32.4 & 2340 & $\mathrm{NE}$ & & $-3.0^{3 \mathrm{a}}$ \\
\hline 73 & A $51 \mathrm{~d} / 10$ & Hüfi & 13.73 & 21.1 & 1740 & SW & & $+5.5^{2 \mathbf{a}}$ \\
\hline 74 & A $51 \mathrm{c} / 2$ & Griess (Unterschächen) & 2.48 & 83.1 & 2160 & $\mathrm{~N}$ & -5.5 & st. \\
\hline 75 & A $51 \mathrm{~h} / 14$ & Firnälpli-Ost & 0.54 & 63.6 & 1880 & N & & $+49.9^{3 a}$ \\
\hline 76 & A $51 \mathrm{~h} / 2$ & Griess (Griessen) & 1.27 & 35.4 & 2420 & W & & $+70^{3 \mathrm{a}}$ \\
\hline
\end{tabular}




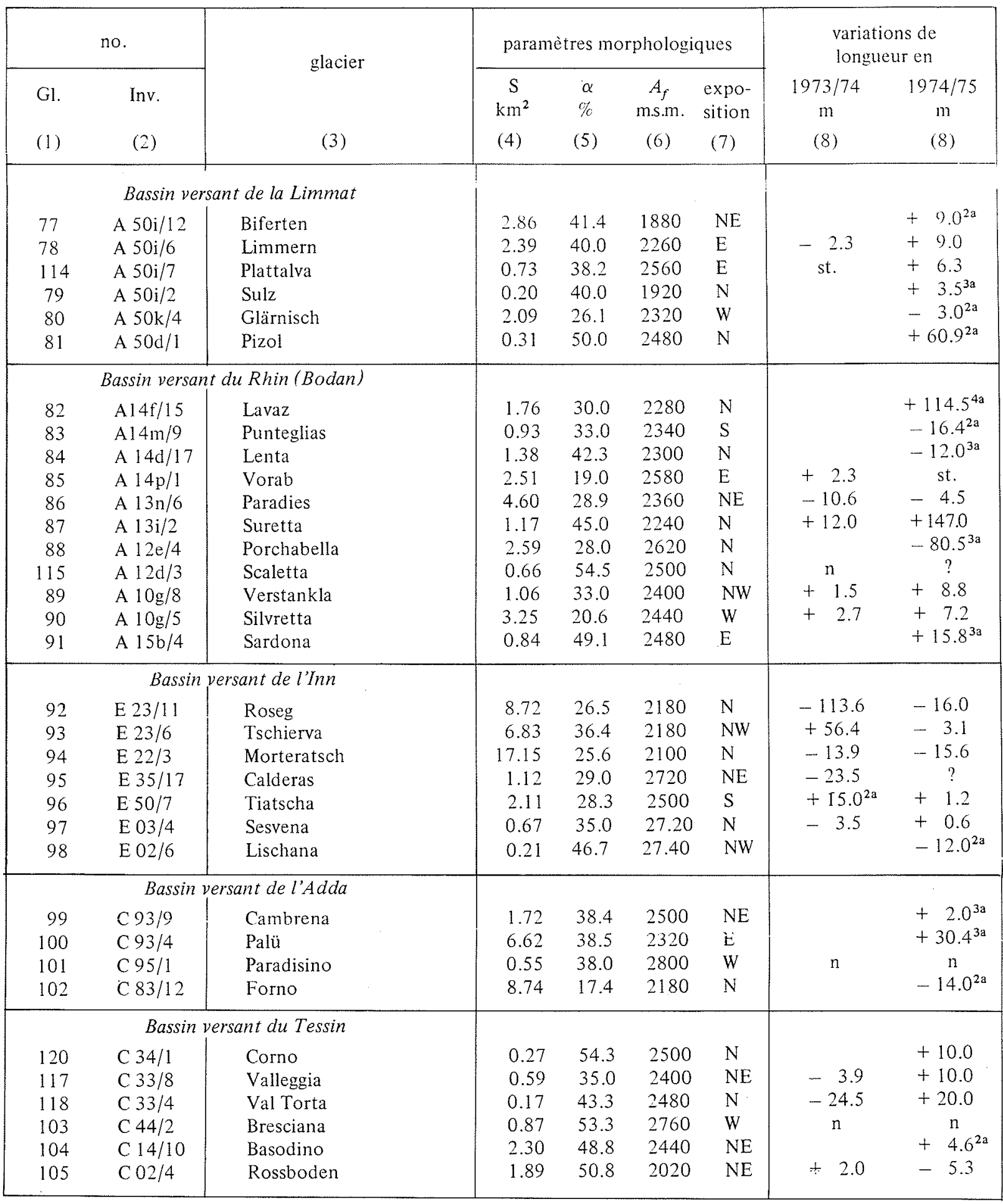

Remarques:

(1) Le $n^{\circ}$ Gl est le numéro du glacier dans le réseau d'observations de la Commission des Glaciers de la Société helvétique des sciences naturelles et des Laboratoires de Recherches hydrauliques, hydrologiques et glaciologiques de l'EPF Zurich. Les $n$ 's Gl. correspondent aux numéros de la figure 1 .

(2) Le $n^{\circ}$ Inv. est le numéro du glacier dans l'inventaire des glaciers suisses élaboré par l'Institut de Géographie de l'EPF Zurich. Les périmètres des glaciers Inv. B 60/9, A 54e/12 et A 54e/13 ne correspondent pas à ceux des glaciers G1 19, 20,53 et 54 .

(3) Nom du glacier dans le réseau Gl.

(4) $S=$ surface du glacier

(5) $\alpha=$ pente moyenne

(6) $A_{f}=$ altitude du front

(7) Exposition de la zone d'ablation

(8) Si la valeur de la variation est valable pour une période de plusieurs années, on a noté le nombre d'années comme suit :

Exemple : Gl. $14:-31.3^{2 a}=$ recul de 31.3 mètres en 2 ans.

st. $=$ stationnaire.

$+=$ en crue

$-=$ en décrue

$\mathrm{x}=$ valeur non chiffrée

$\mathrm{n}=$ non observé

$?=$ résultat incertain 


\begin{tabular}{|c|c|c|c|c|c|c|}
\hline \multicolumn{5}{|c|}{$\begin{array}{l}\text { Variations de longueur des glaciers suisses en 1974/75 } \\
\text { Récapitulation }\end{array}$} & \multicolumn{2}{|c|}{ Tableau $2 a$} \\
\hline \multirow{2}{*}{ Bassin versant } & \multicolumn{6}{|c|}{ Nombre de glaciers } \\
\hline & $\begin{array}{l}\text { du } \\
\text { réseau }\end{array}$ & $\begin{array}{c}\text { non } \\
\text { observés }\end{array}$ & $\begin{array}{l}\text { avec résultat } \\
\text { incertain }\end{array}$ & en crue & stationnaires & $\begin{array}{l}\text { en } \\
\text { décrue }\end{array}$ \\
\hline Rhône & 51 & 1 & 0 & 21 & 5 & 24 \\
\hline Aar & 19 & 3 & 0 & 9 & 2 & 5 \\
\hline Reuss & 11 & 0 & 0 & 9 & 2 & 0 \\
\hline Limmat & 6 & 0 & 0 & 5 & 0 & 1 \\
\hline Rhin (Bodan) & 11 & 0 & 1 & 5 & 1 & 4 \\
\hline $\operatorname{Inn}$ & 7 & 0 & 1 & 1 & 1 & 4 \\
\hline Adda & 4 & 1 & 0 & 2 & 0 & 1 \\
\hline Tessin & 6 & 1 & 0 & 4 & 0 & 1 \\
\hline Total & 115 & $6(1)$ & $2(2)$ & $56(3)$ & $11(4)$ & $40(5)$ \\
\hline
\end{tabular}

Remarques : Les notes (1) à (5) se refèrent aux glaciers qui ont les numéros suivants (v. tableau 1) :

$\begin{array}{rrrrrrrrrrrrrrrr}\text { (1) } & 9 & 56 & 64 & 101 & 103 & 110 & & & & & & & & \\ \text { (2) } & 95 & 115 & & & & & & & & & & & & \\ \text { (3) } & 1 & 2 & 8 & 10 & 11 & 12 & 13 & 19 & 21 & 25 & 26 & 27 & 28 & 37 \\ & 38 & 39 & 41 & 42 & 43 & 47 & 53 & 54 & 57 & 59 & 60 & 61 & 63 & 66 \\ & 67 & 68 & 69 & 70 & 71 & 73 & 75 & 76 & 77 & 78 & 79 & 81 & 82 & 87 \\ & 89 & 90 & 91 & 96 & 99 & 100 & 104 & 107 & 109 & 111 & 114 & 117 & 118 & 120 \\ \text { (4) } & 6 & 7 & 20 & 45 & 46 & 55 & 62 & 72 & 74 & 85 & 97 & & & \\ \text { (5) } & 3 & 4 & 5 & 14 & 15 & 16 & 17 & 18 & 22 & 23 & 24 & 29 & 30 & 31 \\ & 32 & 33 & 34 & 35 & 36 & 40 & 44 & 48 & 49 & 50 & 51 & 52 & 58 & 65 \\ & 80 & 83 & 84 & 86 & 88 & 92 & 93 & 94 & 98 & 102 & 105 & 106 & & \end{array}$

A la figure 2 , la fréquence des variations de longueur des glaciers en $1974 / 75$ est reportée par classes de 5 mètres. On a considéré tous les 101 glaciers dont on dispose de chiffres pour la variation. Dans le cas où on ne connait que la variation totale en plusieurs années, on l'a répartie en parts égales sur chaque année. Les variations d'environ deux tiers des fronts glaciaires sont si- tuées entre - 10 mètres et +10 mètres. La variation moyenne de longueur des 101 glaciers est de $+1,7$ mètres. Cette valeur dévie de celle de + 5,6 mètres indiquéc au tableau 6 a et calculée pour un échantillon de 56 glaciers seulement. Au tableau $6 a$ on avait éliminé tous les résultats influencés par un lac artificiel et tous ceux qui sont valables pour une période de plusieurs années.

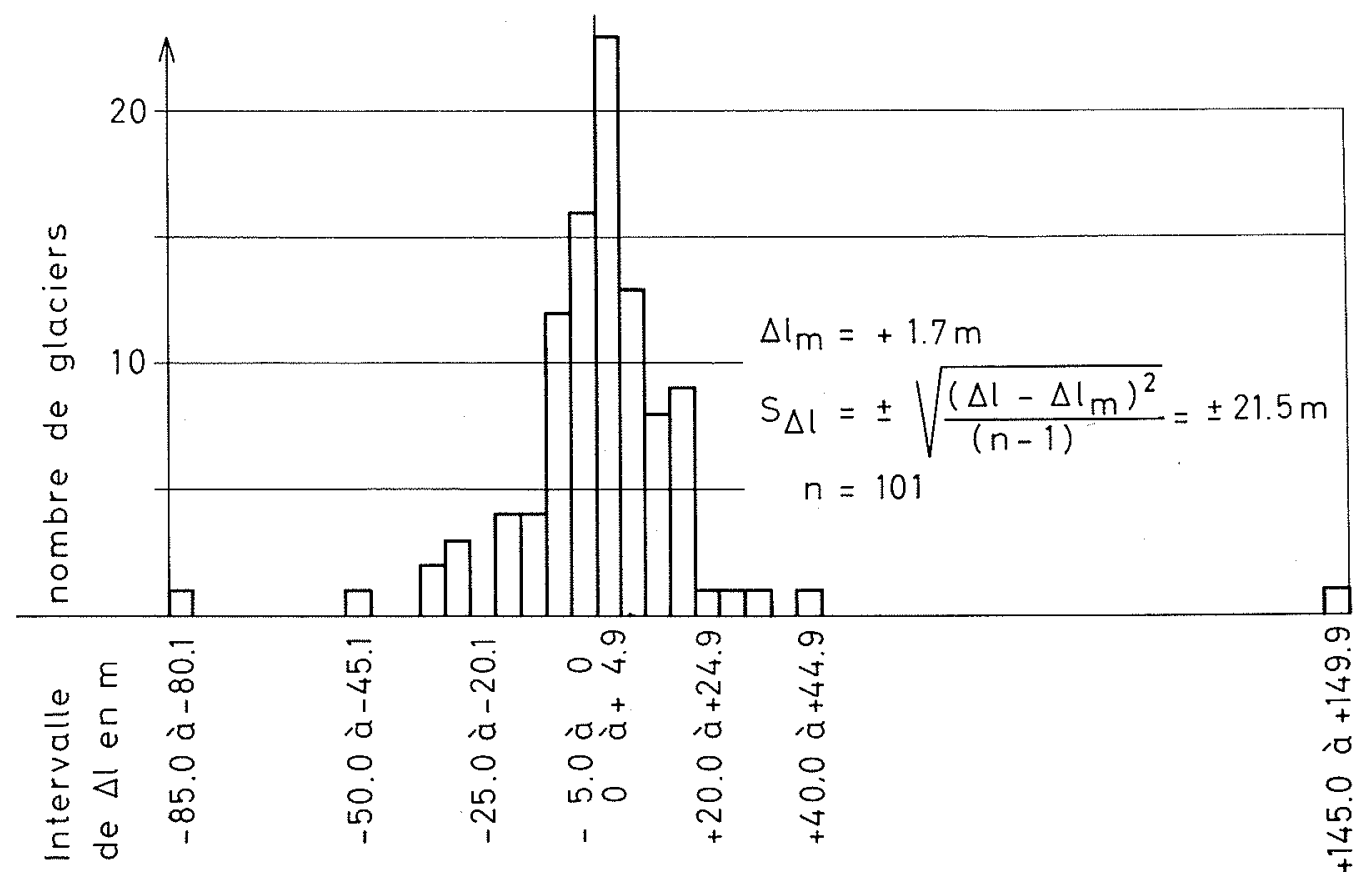

$2 /$ Fréquence des variations $\angle /$ de longueur des glaciers des Alpes suisses en 1974/75 
Les bilans de masse spécifiques annuels de 1974/75 présentés au tableau $2 \mathrm{~b}$ sont tous positifs, avec des valeurs $28,0 \mathrm{gr} \mathrm{cm}^{-2}$ à $79,1 \mathrm{gr} \mathrm{cm}^{-2}$.

\subsection{Conditions climatiques en $1974 / 75$}

La figure 3 reflète l'évolution du temps au cours de l'année, représentée sous forme graphique. Pour Zurich, Locarno-Monti et Jungfraujoch, on a reporté les moyennes journalières de la température avec la courbe des moyennes pluriannuelles ; les hauteurs de pluie sont données pour Zurich, Locarno-Monti, le Säntis et Sion. En outre, les sondages aérologiques de Payerne (à 13 heures) permettent d'indiquer l'altitude de l'isotherme de zéro degré. Les précipations tombent sous forme de neige à peu près à ce niveau.

En été 1974, la période d'ablation des glaciers prit fin en quelques jours avec les vents de l'ouest du 22 au 26 septembre accompagnés de chutes de neige jusqu'au niveau des langues glaciaires.

\begin{tabular}{|c|c|c|c|c|}
\hline \multicolumn{4}{|c|}{ Bilans de masse annuels spécifiques en $\mathrm{g} . \mathrm{cm}^{-2}$, en $1974 / 75$} & Tableau 2b \\
\hline Glacier & $\begin{array}{l}\text { Surface } \\
\text { en } \mathrm{km}^{2}\end{array}$ & $\begin{array}{l}\text { Bilan b } \\
g \cdot \mathrm{cm}^{-2}\end{array}$ & Année de bilan & $\begin{array}{l}\text { Ligne d'équilibre } \\
\text { en m sur mer }\end{array}$ \\
\hline 3 Gries & 6.28 & +28.0 & $18.10 .74-6.10 .75$ & 2720 \\
\hline 5 Aletsch & 128.45 & +55.9 & $1.10 .74-30.09 .75$ & - \\
\hline $\begin{array}{l}78 \text { Limmern et } \\
114 \text { Plattalva }\end{array}$ & 3.29 & +57.3 & $3.09 .74-9.09 .75$ & 2420 \\
\hline 90 Silvretta & 3.15 & +79.1 & $17.09 .74-16.09 .75$ & 2580 \\
\hline
\end{tabular}
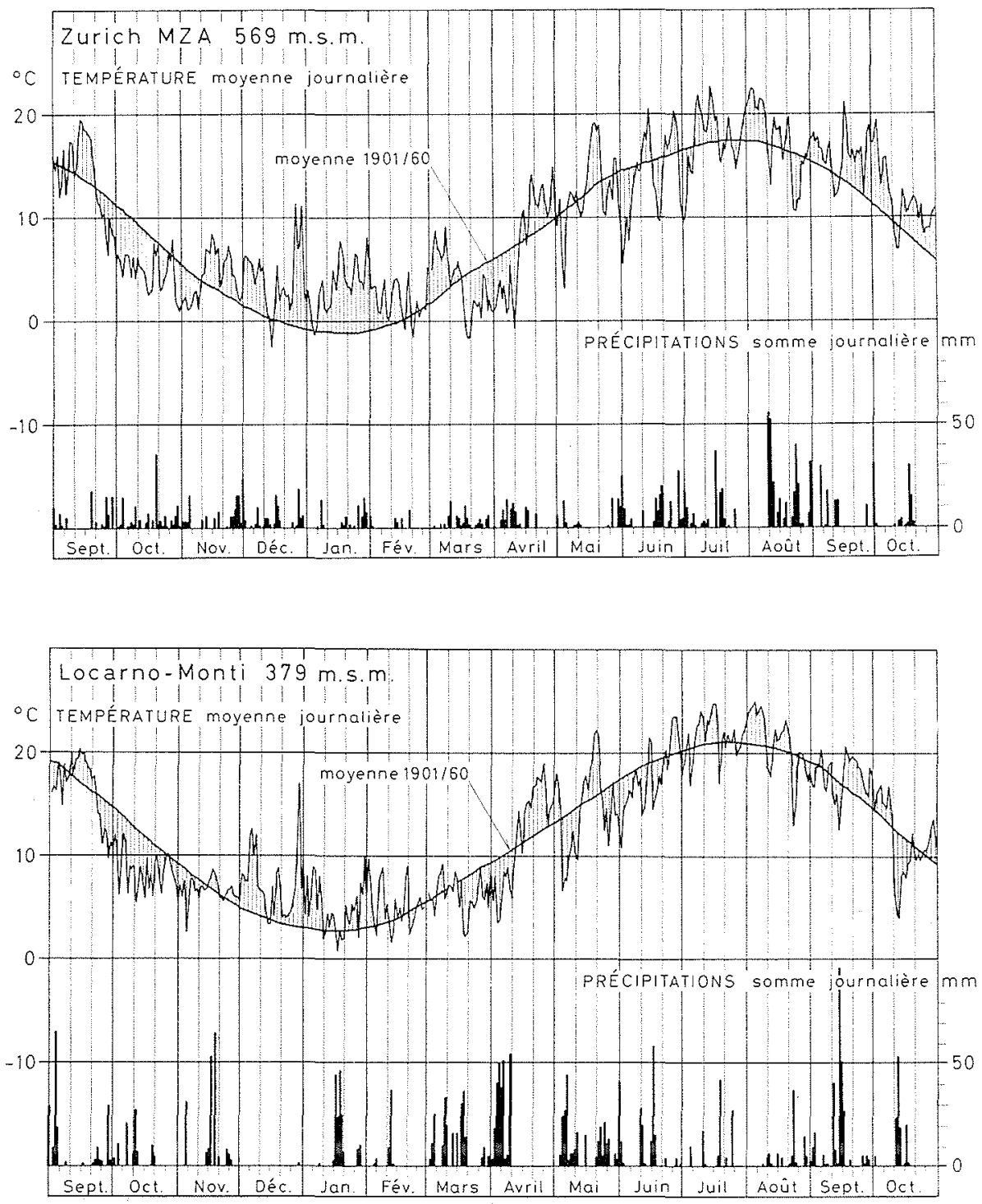

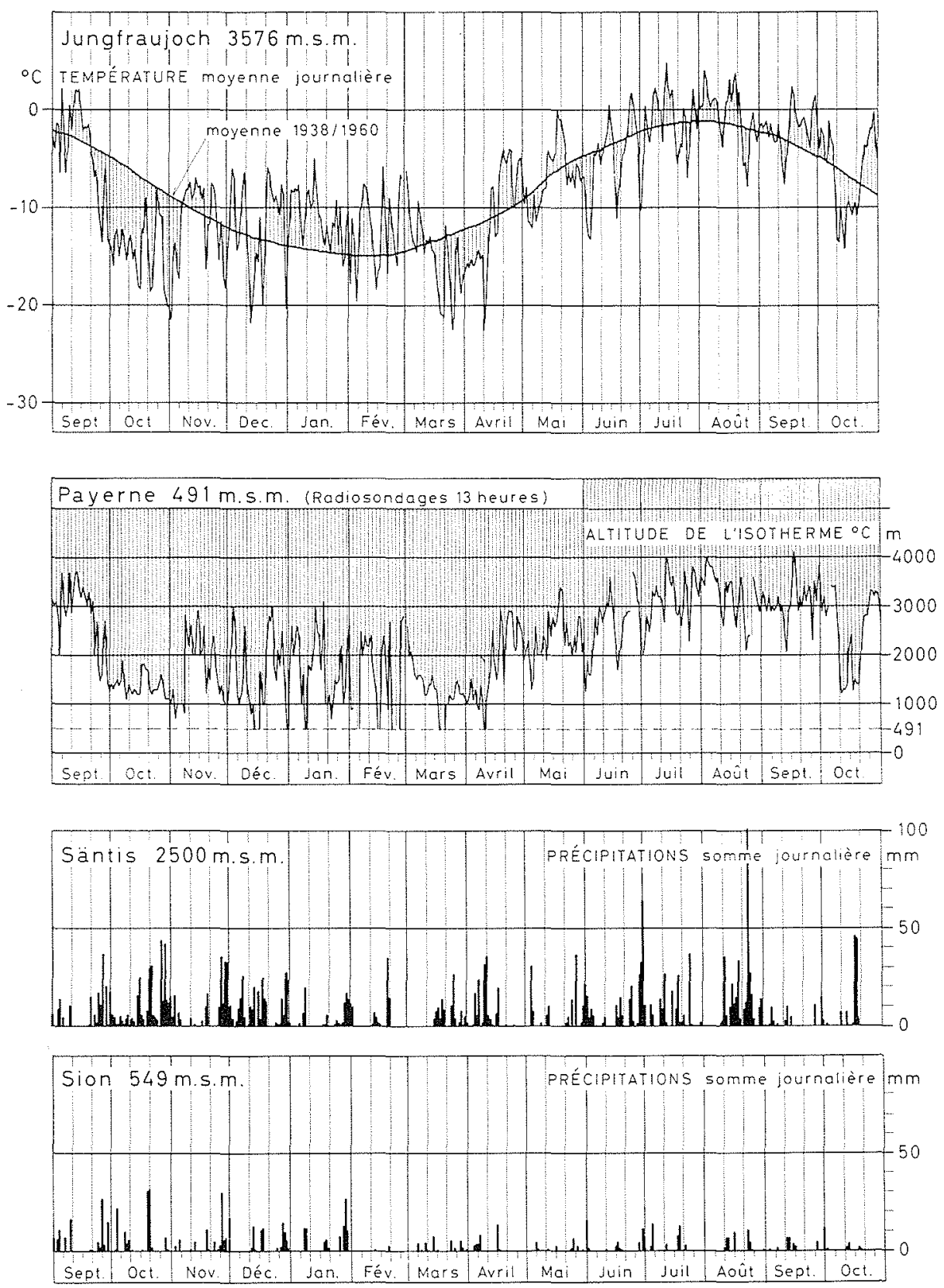
$3 /$ Température (moyenne journalière), précipitations (somme journalière) et altitude de l'isotherme $0^{\circ} \mathrm{C}$ (radiosondages $13 \mathrm{~h}$ ) en
$1974 / 75$

Le mois d'octobre a été excessivement froid partout dans les Alpes suisses. Sauf sur le versant sud, il a été riche en précipitations. Les précipitations cumulées d'octobre 1974 à avril 1975 dépassaient les valeurs normales dans toutes les Alpes suisses. Les chutes de neige abondantes du 3 au 7 avril ont été particulièrement remarquables avec leurs valeurs de neige fraîche cumulée jusqu'à $250 \mathrm{~cm}$ qui sont tombées dans la région centrale des Alpes où elles ont provoqué des avalanches extraordinaires. De mai à septembre 1975 la somme des précipitations ne déviait que peu des valeurs moyennes. La fonte de la couche de neige fut retardée par des chutes de neige fraîche les 3 et 4 mai, au début du mois de juin, les 18,19 et 30 juin. L'enneigement continu au Weissfluhjoch $(2240 \mathrm{~m}$ s.m.) dura 309 jours. Depuis le début de la série d'ob- servations en 1936 cette valeur a été dépassée seulement en $1973 / 74$ avec une durée de 314 jours. Partout dans les Alpes suisses, les températures moyennes de mai à septembre 1975 ne dévièrent que peu des valeurs normales et il y eut quelques périodes chaudes avec une fonte considérable. La période d'ablation de l'été se termina brusquement avec la dépression sur le centre de l'Europe du 10 au 13 octobre avec des chutes de neige sur les versants nord et sud des Alpes jusqu'à environ $600 \mathrm{~m} \mathrm{s.m}$. L'année hydrologique de 1974/75 a été favorable à une augmentation de volume des glaciers suisses. Dans les bassins alpins, la fonte de la couche de neige a compensé plus ou moins le déficit de l'ablation glaciaire, tandis que le débit des bassins sans glaciers dépassait sensiblement les valeurs moyennes. 


\section{Relations entre les variations de lon- gueur et quelques paramètres mor- phologiques}

3.1. Relations entre les variations de longueur et les aires englacées, les pentes moyennes et l'altitude des fronts

En 1974/75, 56 glaciers observés étaient en crue, 40 en décrue. Ces 96 glaciers ont servi de base pour examiner les relations entre le signe de la variation et les aires englacées, respectivement les pentes moyennes des glaciers ou l'altitude des fronts. Les valeurs des paramètres morphologiques des différents glaciers sont inscrites au tableau 1. Dans le tableau $3 \mathrm{a}$, les glaciers sont groupés d'après leur grandeur, 6 classes de 16 glaciers ont été établies. La classe 1 comprend les glaciers les plus petits, la classe 6 les plus grands. Dans chaque classe, on a compte le nombre de glaciers en crue et en décrue. Le même procédé a été appliqué au tableau $3 \mathrm{~b}$ pour les pentes moyennes et au tableau 30 pour l'altitude des fronts. On n'est pas surpris de constater que le nombre de glaciers en crue dépasse le nombre en décrue dans les classes des glaciers petits ou très inclinés. et que le résultat inverse se montre pour les grands glaciers ou pour ceux de faible pente. En ce qui concerne l'altitude des fronts, le tableau $3 c$ ne montre pas de relations évidentes avec les crues et décrues.

Pour un examen statistique on s'est limité à une division en 2 classes par variable, ce qui permet de traiter le problème sans supposer la nature de la loi de distribution. Chacun des 96 glaciers considérés possède les 4 propriétés (variables) signe, surface, pente et altitude du front. On a rangé les signes positifs (les crues) avec la valeur +1 dans la classe 1 , les décrues avec la valeur -1 dans la classe 2 . Des 3 autres variables les grandes valeurs ont été rangées à la classe 1, les petites valeurs à la classe 2. Pour étudier les dépendances totales et partielles entre les variables (problem of association), on a appliqué la méthode des tables de contingence $(2 \times 2 \times 2 \times 2$ table $)$ décrite par

\begin{tabular}{|c|c|c|c|c|c|c|c|}
\hline \multicolumn{7}{|c|}{$\begin{array}{l}\text { Relation entre les variations de longueur des glaciers su } \\
\text { et les aires englacées, les pentes moyennes et l'altitude }\end{array}$} & \multirow[t]{2}{*}{ Tableau 3} \\
\hline 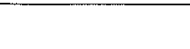 & elation & $1 s-$ air & cées & & & & \\
\hline & \multicolumn{6}{|c|}{$\begin{array}{l}\text { Nombre de glaciers par classe de grandeur } \\
\text { superficie en } \mathrm{km}^{2}\end{array}$} & Total \\
\hline & $\begin{array}{c}0.17 \\
a ̀ \\
0.67\end{array}$ & $\begin{array}{c}0.72 \\
\grave{a} \\
1.86\end{array}$ & $\begin{array}{c}1.89 \\
\grave{a} \\
3.25\end{array}$ & $\begin{array}{c}3.35 \\
\grave{a} \\
6.58\end{array}$ & $\begin{array}{c}6.60 \\
\grave{a} \\
10.89\end{array}$ & $\begin{array}{c}13.70 \\
\grave{a} \\
86.76\end{array}$ & $\begin{array}{c}0.17 \\
\text { à } \\
86.76\end{array}$ \\
\hline classe no & 1 & 2 & 3 & 4 & 5 & 6 & 1 à 6 \\
\hline $\begin{array}{l}\text { en crue } \\
\text { en décrue }\end{array}$ & $\begin{array}{r}11 \\
5\end{array}$ & $\begin{array}{r}11 \\
5\end{array}$ & $\begin{array}{r}13 \\
3\end{array}$ & $\begin{array}{r}10 \\
6\end{array}$ & $\begin{array}{r}6 \\
10\end{array}$ & $\begin{array}{r}5 \\
11\end{array}$ & $\begin{array}{l}56 \\
40\end{array}$ \\
\hline \multirow[t]{4}{*}{ total } & 16 & 16 & 16 & 16 & 16 & 16 & 96 \\
\hline & \multicolumn{7}{|c|}{ b) Relation variations - pentes moyennes } \\
\hline & \multicolumn{6}{|c|}{$\begin{array}{c}\text { Nombre de glaciers par classe de pente } \\
\text { inclinaison en } \%\end{array}$} & Total \\
\hline & $\begin{array}{c}73.1 \\
\grave{a} \\
46.4\end{array}$ & $\begin{array}{c}45.5 \\
\grave{a} \\
38.5\end{array}$ & $\begin{array}{c}38.4 \\
\dot{a} \\
33.0\end{array}$ & $\begin{array}{c}32.9 \\
\grave{a} \\
27.6\end{array}$ & $\begin{array}{c}27.1 \\
\grave{a} \\
21.2\end{array}$ & $\begin{array}{c}21.1 \\
\grave{a} \\
10.6\end{array}$ & $\begin{array}{c}73.1 \\
\grave{a} \\
10.6\end{array}$ \\
\hline classe no & 1 & 2 & 3 & 4 & 5 & 6 & 1 à 6 \\
\hline $\begin{array}{l}\text { en crue } \\
\text { en décrue }\end{array}$ & $\begin{array}{r}10 \\
6\end{array}$ & $\begin{array}{r}13 \\
3\end{array}$ & $\begin{array}{r}12 \\
4\end{array}$ & $\begin{array}{r}10 \\
6\end{array}$ & $\begin{array}{l}7 \\
9\end{array}$ & $\begin{array}{r}4 \\
12\end{array}$ & $\begin{array}{l}56 \\
40\end{array}$ \\
\hline \multirow[t]{4}{*}{ total } & 16 & 16 & 16 & 16 & 16 & 16 & 96 \\
\hline & \multicolumn{7}{|c|}{ c) Relation variations - altitude des fronts } \\
\hline & \multicolumn{6}{|c|}{$\begin{array}{l}\text { Nombre de glaciers par classe d'altitude du front } \\
\text { altitude en metres sur mer }\end{array}$} & Total \\
\hline & $\begin{array}{c}2840 \\
\grave{a} \\
2560\end{array}$ & $\begin{array}{c}2540 \\
\dot{a} \\
2440\end{array}$ & $\begin{array}{c}2420 \\
\grave{a} \\
2320\end{array}$ & $\begin{array}{c}2320 \\
\text { à } \\
2180\end{array}$ & $\begin{array}{c}2180 \\
\dot{a} \\
2020\end{array}$ & $\begin{array}{c}2000 \\
\grave{a} \\
1240\end{array}$ & $\begin{array}{c}2840 \\
\mathrm{a} \\
1240\end{array}$ \\
\hline classe no & 1 & 2 & 3 & 4 & 5 & 6 & 1 à 6 \\
\hline $\begin{array}{l}\text { en crue } \\
\text { en décrue }\end{array}$ & $\begin{array}{l}9 \\
7\end{array}$ & $\begin{array}{r}11 \\
5\end{array}$ & $\begin{array}{l}7 \\
9\end{array}$ & $\begin{array}{r}10 \\
6\end{array}$ & $\begin{array}{l}8 \\
8\end{array}$ & $\begin{array}{r}11 \\
5\end{array}$ & $\begin{array}{l}56 \\
40\end{array}$ \\
\hline total & 16 & 16 & 16 & 16 & 16 & 16 & 96 \\
\hline
\end{tabular}


exemple dans le chapitre "categorized data" dans M.G. Kendall and A. Stuart : "The Advanced Theory of Statistics", vol. 2, p. 538 - 556, Griffin, London 1961. D'après cette théorie, on a calculé pour les différents cas les coefficients d'association $Q$ et, avec un test unilatéral, la probabilité de l'erreur $(1-s)$ de première espèce appartenant à $Q$ ou le niveau de signification (significance level) $\mathrm{s}$ de $\mathrm{Q}$. Les coefficients d'association et leur niveau de signification $s$ ont été évalués avec différentes limites entre les classes 1 et 2 des variables surface $S$, pente $\alpha$ et altitude $H$. Les chiffres qui accompagent entre parenthèses les résultats mentionnés ci-dessous de $\mathrm{s}$ signifient le nombre de glaciers rangés dans la classe 1 de la variable indiquée. Le coefficient d'association entre la surface $S$ et le signe est significatif au niveau de $s=99,76 \%$ (S 48) et de $97,88 \%$ (S 40). Si l'on ne considère que les fronts à basse altitude, les valeurs de $\mathrm{s}$ sont de $98,80 \%$ pour (S 48, H 48) et également de $98,80 \%$ pour ( $\mathrm{S} 40 . \mathrm{H} 48$ ). Pour l'échantillon qui ne comprend que les fronts à haute altitude, le cas ( $\mathrm{S} 48, \mathrm{H} 48$ ) est significatif avec $\mathrm{s}=96,74 \%$. La dépendance entre la pente $\alpha$ et le signe est significative au niveau $\mathrm{s}$ de $99,76 \%(\alpha 48)$ et de $99,80 \%(\alpha 56)$. Si l'on ne considère que les fronts à haute altitude, s est de $87,00 \%(\alpha 48, \mathrm{H} \mathrm{48})$ et de $94,78 \%(\alpha$ 56, H 48). Pour les fronts à basse altitude, le coefficient d'association entre la pente et le signe est assuré avec $s=99,43 \%(\alpha 48, \mathrm{H} 48)$ et $\mathrm{s}=98,42 \%$ ( $\alpha$ 56, H 48). La dépendance entre l'altitude $H$ du front et le signe n'est significative que dans les cas où l'on se borne aux petits glaciers avec $\mathrm{s}=86,50 \%(\mathrm{H} \mathrm{48,S} \mathrm{48)} \mathrm{et}$ $\mathrm{s}=92,00 \%(\mathrm{H} \mathrm{48,S} \mathrm{40)} \mathrm{ou} \mathrm{aux} \mathrm{glaciers} \mathrm{à} \mathrm{la} \mathrm{fois} \mathrm{petits}$ et de forte pente avec s $=87,50 \%(\mathrm{H} 48, \mathrm{~S} \mathrm{40}, \alpha 56)$. La dépendance entre surface et pente est significative à presque $100 \%$ ( $\mathrm{S} \mathrm{48,} \mathrm{H} \mathrm{48)} \mathrm{ou} \mathrm{(} 40, \mathrm{H} \mathrm{48)}$ ), celle entre pente et altitude du front n'est pas du tout significative. Par contre, la relation entre surface des glaciers et altitude des fronts est significative à un niveau très élevé.

Toutes ces relations sont valables pour les variations de longueur des glaciers suisses observés en 1974/75. Dans ce sens, l'étude présentée ici n'est qu'un essai de décrire les variations d'une année donnée. L'examen des relations pour d'autres années pourrait contribuer à la recherche des facteurs qui conditionnent les variations. Un autre moyen dans ce but serait l'étude des glaciers de comportement exceptionnel qui n'obéissent pas aux règles générales.

\subsection{Relations entres les variations de longueur et l'expo-} sitions

La distribution du nombre de glaciers en crue, stationnaires et en décrue sur les 8 expositions $N, N E, \ldots \ldots$,
NW est représentée au tableau 4 et à la figure 4. L'exposition des différents glaciers reportée dans le tableau 1 a été déterminée pour les zones d'ablation. On a examiné les 107 glaciers dont on connait pour 1974/75 le signe de la variation de longueur. Environ un tiers des langues glaciaires observées est exposé au nord, a peu près la moitié se répartit sur les trois expositions de NE, E et NW, tandis que chacune des quatre directions $S E, S, S W$ et W ne comprend que 5 glaciers.

La question se pose si le quotient $+/-$ du nombre de glaciers en crue et en décrue dépend de l'exposition. Une difficulté d'en décider réside dans le fait que les quotients pour les directions SE, S, SW et W ne sont guère significatifs, étant donné que les échantillons de ces expositions sont très petits. En outre, on peut attribuer parfois l'exposition d'un glacier aussi bien à une direction qu'à une des

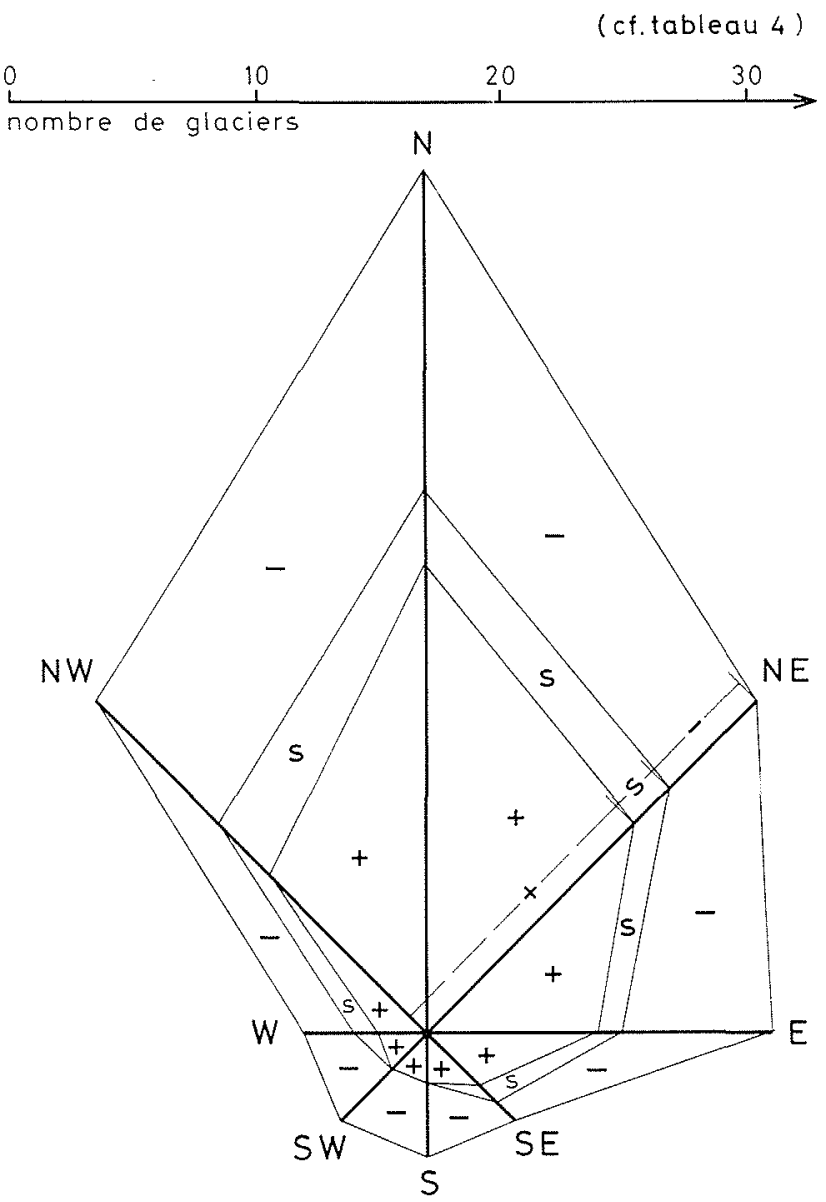

4/ Nombre de glaciers en crue ( +1 , stationnaires (s) et en décrue (-) dans les différentes expositions (année 1974/75)

\begin{tabular}{|c|c|c|c|c|c|c|c|c|c|}
\hline \multicolumn{9}{|c|}{ Relations entre les variations de longueur des glaciers en $1974 / 75$ et l'exposition } & Tableau 4 \\
\hline & \multicolumn{8}{|c|}{ nombre de glaciers par exposition } & \multirow[b]{2}{*}{ Total } \\
\hline & $\mathrm{N}$ & $\mathrm{NE}$ & $\mathrm{E}$ & $\mathrm{SE}$ & $\mathrm{S}$ & SW & W & NW & \\
\hline en crue & 19 & 12 & 7 & 3 & 2 & 2 & 2 & 9 & 56 \\
\hline stationnaire & 3 & 2 & 1 & 1 & 0 & 0 & 1 & 3 & 11 \\
\hline en décrue & 13 & 5 & 6 & 1 & 3 & 3 & 2 & 7 & 40 \\
\hline Total & 35 & 19 & 14 & 5 & 5 & 5 & 5 & 19 & 107 \\
\hline
\end{tabular}


directions voisines. Pour ces raisons on a procédé à un lissage en remplaçant les directions par les secteurs. Ceux-ci sont nommés par leur direction centrale et délimités par les deux directions voisines. Pour déterminer le nombre de glaciers en crue ou en décrue par secteur, on a additionné le nombre de glaciers dans les trois directions du secteur, en prenant le poids 1 pour la direction centrale et le poids 0,5 pour les directions marginales du secteur.

Le quotient $+/$-des sommes pondérées du nombre de glaciers en crue et de glaciers en décrue par secteur est noté au tableau 5 et dessiné à la figure 5 pour les observations de l'année 1974/75. Il est exempt de doute que le quotient varie d'une façon systématique avec l'exposition, montrant un maximum au secteur NE et un minimum au secteur SW. Les crues dominent donc dans le secteur NE et dans les secteurs voisins, les décrues se trouvent de préférence aux secteurs $\mathrm{SW}$ et $\mathrm{S}$. Ce résultat est évident, mais pas facile à expliquer. Est-ce qu'il y a une concentration de glaciers exposés au nord dans une région, où pendant les dernières années, les conditions climatiques étaient particuliérement favorables aux crues? Les conditions climatiques de la dernière décennie ont-elles favorisé une avance des glaciers exposés au nord ? On pourrait penser à une fréquence élevée de chutes de neige précoces en été qui ont terminé et raccourci d'une façon extrême la période d'ablation sur les versants nord, tandis que la fonte a fait disparaitre la neige fraîche relativement vite aux versants sud, en permettant une continuation importante de la période d'ablation en automne. Quelles sont les conséquences de la longue période de récession des glaciers pour la situation actuelle ? La relation constatée entre le quotient $+/-$ et l'exposition est-elle le résultat de raisons morphologiques ou climatiques ou d'un mécanisme complexe? L'analyse approfondie reste à faire.

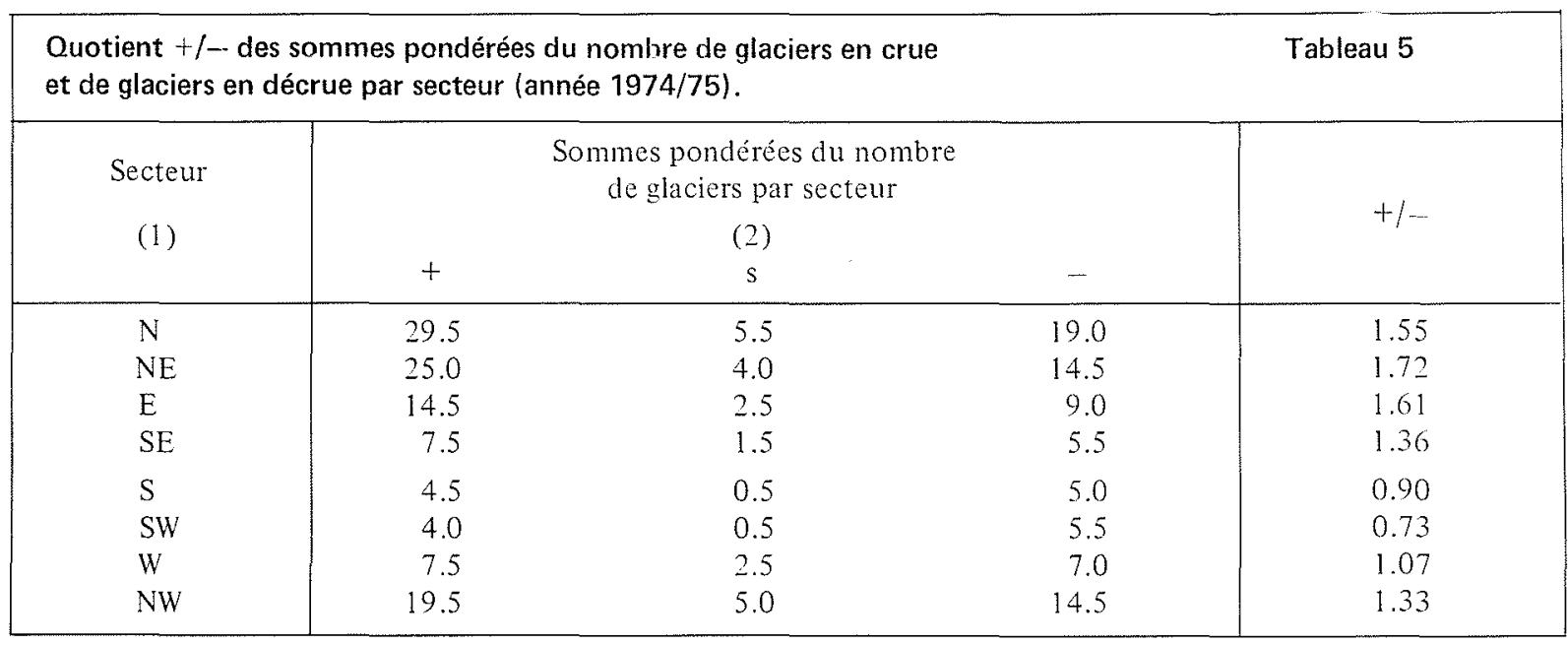

(1) Le secteur est nommé par sa direction centrale et il est délimité par les deux directions voisines (le secteur NW par exemple est délimité par les directions marginales de $\mathrm{W}$ et $\mathrm{N}$ ).

(2) On a additionné le nombre de glaciers exposés dans les trois directions du secteur, en prenant le poids 1 pour la direction centrale et le poids 0.5 pour les deux directions marginales du secteur.

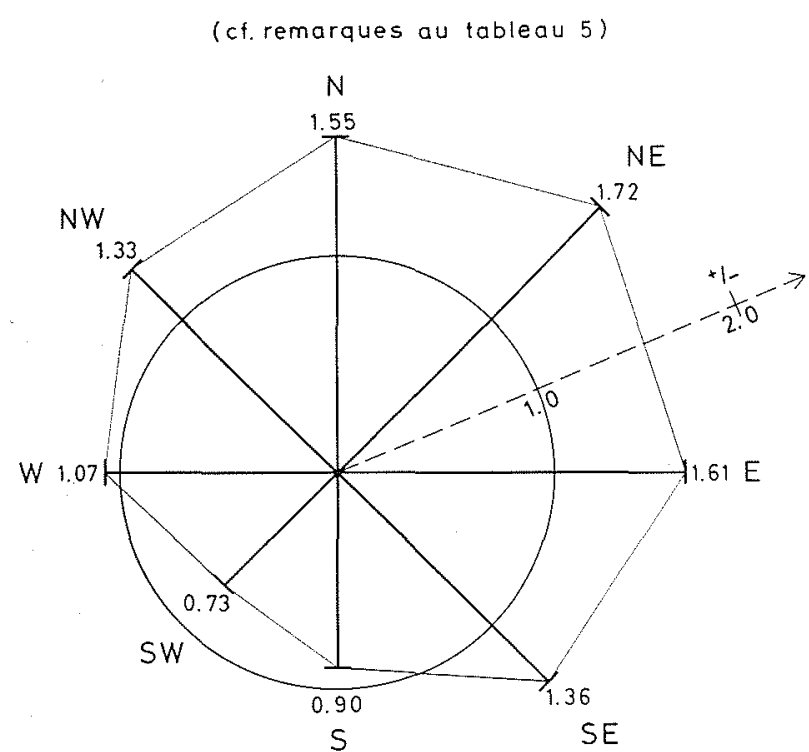

$5 /$ Quotient $+1-$ du nombre de glaciers en crue et de glaciers en décrue pour les différents secteurs (année 1974/75)

\section{Les variations des glaciers suisses pen- dant la Décennie Hydrologique Inter- nationale de $1964 / 65$ à $1973-74$.}

Les variations de fronts des glaciers sont le résultat d'une part de la fonte et de l'accumulation aux fronts dans l'année hydrologique considérée, d'autre part du mouvement qui dépend des bilans spécifiques de masse durant une série d'années. Dans le cas de crues extrêmes, le phénomène complexe des "surges" est également à considérer. Pour mieux comprendre le comportement des glaciers dans une année d'observation donnée, il est nécessaire de connaître l'évolution des glaciers dans les années précédentes. A cette fin, les variations de longueur et les bilans ainsi que quelques informations climatiques sont assemblées aux tableaux 6 et 8 et aux figures 6 et 7 .

Le pourcentage des glaciers en crue, stationnaires et en décrue est reporté au tableau 6 a pour les années de $1963 / 64$ à 1974/75 et à la figure 7 à partir de $1891 / 92$. On constate que 1963/64 était la demière année d'une 


\begin{tabular}{|c|c|c|c|c|c|c|c|c|c|c|}
\hline \multicolumn{9}{|c|}{$\begin{array}{l}\text { Nombre de glaciers en crue, stationnaires et en décrue, en pourcent } \\
\text { du nombre total des glaciers observés ainsi que les variations moyennes } \\
\text { de longueur de } 1963 / 64 \text { à } 1974 / 75 \text {. }\end{array}$} & \multicolumn{2}{|c|}{ Tableau 6a } \\
\hline \multirow{2}{*}{ Année } & \multicolumn{5}{|c|}{ nombre de glaciers } & \multicolumn{3}{|c|}{$\begin{array}{c}\text { \% des glaciers } \\
\text { observés }\end{array}$} & \multicolumn{2}{|c|}{$\begin{array}{l}\text { variations moyen- } \\
\text { nes } \Delta l_{m} \text { de } \\
\text { longueur }\end{array}$} \\
\hline & $\begin{array}{l}\text { dans } \\
\text { le ré- } \\
\text { seau }\end{array}$ & $\begin{array}{l}\text { obser- } \\
\text { vés } \\
100 \%\end{array}$ & $\begin{array}{l}\text { en } \\
\text { crue } \\
+\end{array}$ & $\begin{array}{l}\text { station- } \\
\text { naires } \\
\text { s }\end{array}$ & $\begin{array}{c}\text { en } \\
\text { décrue } \\
-\end{array}$ & $\begin{array}{c}\text { en } \\
\text { crue } \\
+\end{array}$ & $\begin{array}{l}\text { station- } \\
\text { naires } \\
\text { s }\end{array}$ & $\begin{array}{c}\text { en } \\
\text { décrue } \\
-\end{array}$ & $\begin{array}{c}\text { nombre } \\
\text { de gla- } \\
\text { ciers }\end{array}$ & $\begin{array}{c}\Delta l_{m} \\
\mathrm{~m}\end{array}$ \\
\hline $1963 / 64$ & 105 & 88 & 5 & 1 & 82 & 5.7 & 1.1 & 93.2 & 76 & -14.4 \\
\hline $1964 / 65$ & 105 & 90 & 22 & 10 & 58 & 24.4 & 11.1 & 64.5 & 72 & -10.2 \\
\hline $1965 / 66$ & 105 & 91 & 34 & 3 & 54 & 37.3 & 3.3 & 59.4 & 77 & -2.9 \\
\hline $1966 / 67$ & 105 & 100 & 23 & 3 & 74 & 23.0 & 3.0 & 74.0 & 84 & -7.2 \\
\hline $1967 / 68$ & 105 & 98 & 35 & 6 & 57 & 35.7 & 6.1 & 58.2 & 89 & -0.4 \\
\hline $1968 / 69$ & 105 & 103 & 28 & 5 & 70 & 27.2 & 4.8 & 68.0 & 92 & -7.2 \\
\hline $1969 / 70$ & 105 & 98 & 31 & 6 & 61 & 31.6 & 6.1 & 62.3 & 89 & -1.3 \\
\hline $1970 / 71$ & 110 & 108 & 17 & 5 & 86 & 15.8 & 4.6 & 79.6 & 92 & -10.2 \\
\hline $1971 / 72$ & 114 & 100 & 39 & 7 & 54 & 39.0 & 7.0 & 54.0 & 91 & -2.7 \\
\hline $1972 / 73$ & 114 & 95 & 24 & 8 & 63 & 25.3 & 8.4 & 66.3 & 78 & -10.4 \\
\hline $1973 / 74$ & 115 & 76 & 30 & 14 & 32 & 39.5 & 18.4 & 42.1 & 65 & -1.8 \\
\hline $1974 / 75$ & 115 & 107 & 56 & 11 & 40 & 52.3 & 10.3 & 37.4 & 56 & +5.6 \\
\hline
\end{tabular}

\begin{tabular}{|c|c|c|c|c|c|}
\hline \multicolumn{5}{|c|}{ Les crues des années 1963/64 à 1974/75. } & \multirow[t]{2}{*}{ Tableau 6b } \\
\hline \multirow[b]{2}{*}{ Année } & \multicolumn{4}{|c|}{ Nombre de crues } & \\
\hline & totales & $\begin{array}{c}\text { "vraies" } \\
\text { (1) }\end{array}$ & $\begin{array}{c}\text { diverses } \\
\text { (2) }\end{array}$ & $\begin{array}{c}\text { par allongement } \\
\text { (3) }\end{array}$ & $\begin{array}{c}\text { de fronts enneiges } \\
\text { (4) }\end{array}$ \\
\hline $1963 / 64$ & 5 & 5 & 0 & 0 & 0 \\
\hline $1964 / 65$ & 22 & 12 & 5 & 0 & 5 \\
\hline $1965 / 66$ & 34 & 17 & 2 & 14 & 1 \\
\hline $1966 / 67$ & 23 & 18 & 1 & 4 & 0 \\
\hline $1967 / 68$ & 35 & 26 & 4 & 3 & 2 \\
\hline $1968 / 69$ & 28 & 25 & 1 & 2 & 0 \\
\hline $1969 / 70$ & 31 & 24 & 0 & 4 & 3 \\
\hline $1970 / 71$ & 17 & 17 & 0 & 0 & 0 \\
\hline $1971 / 72$ & 39 & 34 & 0 & 3 & 2 \\
\hline $1972 / 73$ & 24 & 23 & 0 & 1 & 0 \\
\hline $1973 / 74$ & 30 & 27 & 0 & 1 & 2 \\
\hline $1974 / 75$ & 56 & 44 & 1 & 6 & 5 \\
\hline
\end{tabular}

\section{Remarques :}

(1) Avance due surtout au mouvement.

(2) Crue due à un mécanisme inconnu.

(3) Allongement dû à la formation de cônes d'avalanches ou aux accumulations péremes devant le front.

(4) Avance d'une langue enneigée.

longue période de récession excessive et qu'avec 1964/65, soit le début de la Décennie Hydrologique Internationale (DHI), un rétablissement des glaciers se manifeste par un nombre croissant de glaciers en crue. L'évolution dans le temps est encore mieux visible au tableau 6 b et à la figure 6 , où les crues sont classées d'après leur mécanisme. Si l'on ne considere que les "vraies" crues dues surtout au mouvement, l'augmentation du nombre de crues est encore plus évidente. Mais ce fait ne permet pas de conclure qu'une crue générale ait commencé ou même que les glaciers soient actuellement plus ou moins en équilibre avec le climat. La comparaison des variations moyennes de longueur du tableau 6 a avec la fréquence des variations reportée à la figure 2 montre qu'une seule année hostile a la croissance des glaciers suffit pour diminuer sensiblement le nombre de glaciers en crue. 


\begin{tabular}{|c|c|c|c|c|c|c|c|c|}
\hline \multicolumn{7}{|c|}{ Bilans annuels spécifiques de masse $\bar{b} 1963 / 64$ à 1974/75. } & \multicolumn{2}{|c|}{ Tableau 7} \\
\hline & $\begin{array}{c}\text { Sarennes } \\
\mathrm{Sa}\left({ }^{\mathbf{l}}\right)\end{array}$ & $\begin{array}{l}\text { Aletscly } \\
5\left({ }^{2}\right)\end{array}$ & $\begin{array}{l}\text { Gries } \\
3\left({ }^{2}\right)\end{array}$ & $\begin{array}{c}\text { Limmern }\left({ }^{5}\right) \\
78\left({ }^{2}\right)\end{array}$ & $\begin{array}{l}\text { Silvretta } \\
90\left({ }^{2}\right)\end{array}$ & $\begin{array}{c}\text { Hintereis } \\
\mathrm{H}^{x}\left({ }^{3}\right)\end{array}$ & $\begin{array}{c}\text { Kessel- } \\
\text { wand } \\
K\left({ }^{3}\right)\end{array}$ & $\begin{array}{c}\text { Stub. } \\
\text { Sonnbl. } \\
\text { StS }\left(^{4}\right)\end{array}$ \\
\hline & $\mathrm{kg} \mathrm{m}-2$ & $\mathrm{~kg} \mathrm{~m}^{-2}$ & $\mathrm{~kg} \mathrm{~m}^{-2}$ & $\mathrm{~kg} \mathrm{~m}^{-2}$ & $\mathrm{~kg} \mathrm{~m}^{-2}$ & $\mathrm{~kg} \mathrm{~m} \mathrm{~m}^{-2}$ & $\mathrm{~kg} \mathrm{~m}{ }^{-2}$ & $\mathrm{~kg} \mathrm{~m}^{-2}$ \\
\hline $1963 / 64$ & -1820 & -1269 & $-\quad 862$ & -1833 & -1365 & -1244 & -540 & -932 \\
\hline $1964 / 65$ & 30 & 1178 & 925 & 924 & 1295 & 925 & 1040 & 1976 \\
\hline $1965 / 66$ & 420 & 620 & $-\quad 279$ & 505 & 1174 & 344 & 590 & 736 \\
\hline $1966 / 67$ & -410 & 296 & 260 & -363 & 340 & 20 & 300 & 160 \\
\hline $1967 / 68$ & 340 & 668 & 332 & 397 & 616 & 338 & 460 & 236 \\
\hline $1968 / 69$ & -360 & 308 & 269 & -140 & -251 & -430 & -150 & -247 \\
\hline $1969 / 70$ & -410 & $-\quad 124$ & $\begin{array}{l}-519 \\
\end{array}$ & -158 & 125 & $-\quad 550$ & 0 & 144 \\
\hline $1970 / 71$ & -1110 & -705 & -1069 & -1336 & -887 & -600 & 50 & $-\quad 392$ \\
\hline $1971 / 72$ & -370 & -218 & 446 & -245 & -256 & 70 & 370 & 128 \\
\hline $1972 / 73$ & -670 & -529 & -1116 & -1010 & -1213 & -1230 & $-\quad 380$ & -721 \\
\hline $1973 / 74$ & -1600 & 70 & -159 & 85 & 744 & 60 & 570 & 576 \\
\hline $1974 / 75$ & 110 & 559 & 280 & 573 & 791 & 60 & 370 & 397 \\
\hline
\end{tabular}

Surface englacée en $\mathrm{km}^{2}$ :

\begin{tabular}{|lrr|lrc|}
\hline \multicolumn{1}{|c|}{ Glacier } & année & $\mathrm{km}^{2}$ & & Glacier & année \\
\hline Sarennes & 1950 & 0.82 & Silvretta & 1956 & 3.33 \\
Aletsch & 1927 & 137.90 & & 1973 & 3.09 \\
(bassin total de la Massa) & 1957 & 129.76 & Hintereis & 1964 & 9.06 \\
& 1964 & 129.40 & & 1975 & 8.97 \\
& 1975 & 128.45 & Kesselwand & 1964 & 4.06 \\
Gries & 1961 & 6.69 & & 1973 & 4.26 \\
& 1971 & 6.30 & Stubacher Sonnblickkees & 1964 & 1.26 \\
Limmem (Plattalva 114, inclus) & 1959 & 3.29 & & 1974 & 1.76 \\
\hline
\end{tabular}

(1) Rapports de M. Louis de Crécy, Grenoble, aux Séances de la SHF, Section de Glaciologie, 1964 à 1975, et communication personnelle en 1976.

(2) Laboratoires de Recherches hydraulique, hy drologique et glaciologique de l'EPF à Zurich

(3) H. Hoinkes : Zeitschrift für Gletscherkunde und Glazialgeologie, Band VI, Innsbruck 1970.

H. Hoinkes, F. Dreiseitl und H.P. Wagner : "Mass balance of Hintereisferner and Kesselwandferner 1963/64 to 1972/83", IHDActivities in Austria 1965-1974, Vienna 1974

Communication personnelle de G. Markl, Institut für Meteorologie und Geophysik der Universität Innsbruck, en 1976.

(4) Stubacher Sonnblickkees. Communication personnelle de Heinz Slupetzki, Geographisches Institut der Universität Salzburg, en 1976.

(5) Plattalva 114 inclus.

Les variations de longueur sont une information utile pour caractériser l'évolution dans la période d'observation écoulée. Par contre, les bilans annuels spécifiques de masse présentés dans le tableau 7 sont en méme temps efficaces pour l'évolution à long terme. Dans le tableau 7 , les bilans de 4 glaciers suisses sont reportés et comparés avec les résultats du glacier de Sarennes dans les Alpes françaises et de 3 glaciers des Alpes autrichiennes. La cumulation des bilans annuels de la DHI donne les valeurs suivantes:

$\begin{array}{ll}\text { Sarennes } & -414 \mathrm{gr} \mathrm{cm}^{-2} \\ \text { Aletsch } & +156 \mathrm{gr} \mathrm{cm}^{-2} \\ \text { Gries } & -91 \mathrm{gr} \mathrm{cm}^{-2} \\ \text { Limmern } & -134 \mathrm{gr} \mathrm{cm}^{-2} \\ \text { Silvretta } & +169 \mathrm{gr} \mathrm{cm} \\ \text { Hintereis } & -119 \mathrm{gr} \mathrm{cm}^{-2} \\ \text { Kesselwand } & +285 \mathrm{gr} \mathrm{cm}^{-2} \\ \text { Stubacher } & \\ \text { Sonnblickkees } & +260 \mathrm{gr} \mathrm{cm}^{-2}\end{array}$

Les bilans des quatre glaciers suisses cumulés sur 10 ans ne dévient que de peu de l'état stationnaire. Pour les an- nées individuelles, les différences de bilans des glaciers sont compatibles avec les données climatiques des stations voisines présentées sous forme de variables centrées réduites au tableau 8. La conclusion est que pendant la DHI les glaciers des Alpes suisses étaient plus ou moins en équilibre avec le climat, malgré les variations moyennes de longueur des glaciers toujours négatives. Les différences de bilan des glaciers très voisins du Hintereis et du Kesselwand dans le Oetztal sont une indication pour le fait qu'il faut prendre en considération également les propriétés morphologiques responsables pour les temps de réponse des variations des fronts aux bilans. D'après les valeurs de bilans du tableau $6 \mathrm{a}$, on pourrait supposer que pendant la DHI les conditions climatiques étaient plus favorables aux glaciers dans les Alpes orientales que dans les Alpes occidentales, toujours sous réserve de l'influence des parametres morphologiques qui n'est pas suffisamment étudiée.

A la figure 7 , on a reporté les valeurs annuelles des précipitations et de l'écoulement du bassin de la Massa (glaciers d'Aletsch), des bilans spécifiques de masse de huit glaciers ainsi que le pourcentage des glaciers en crue, 


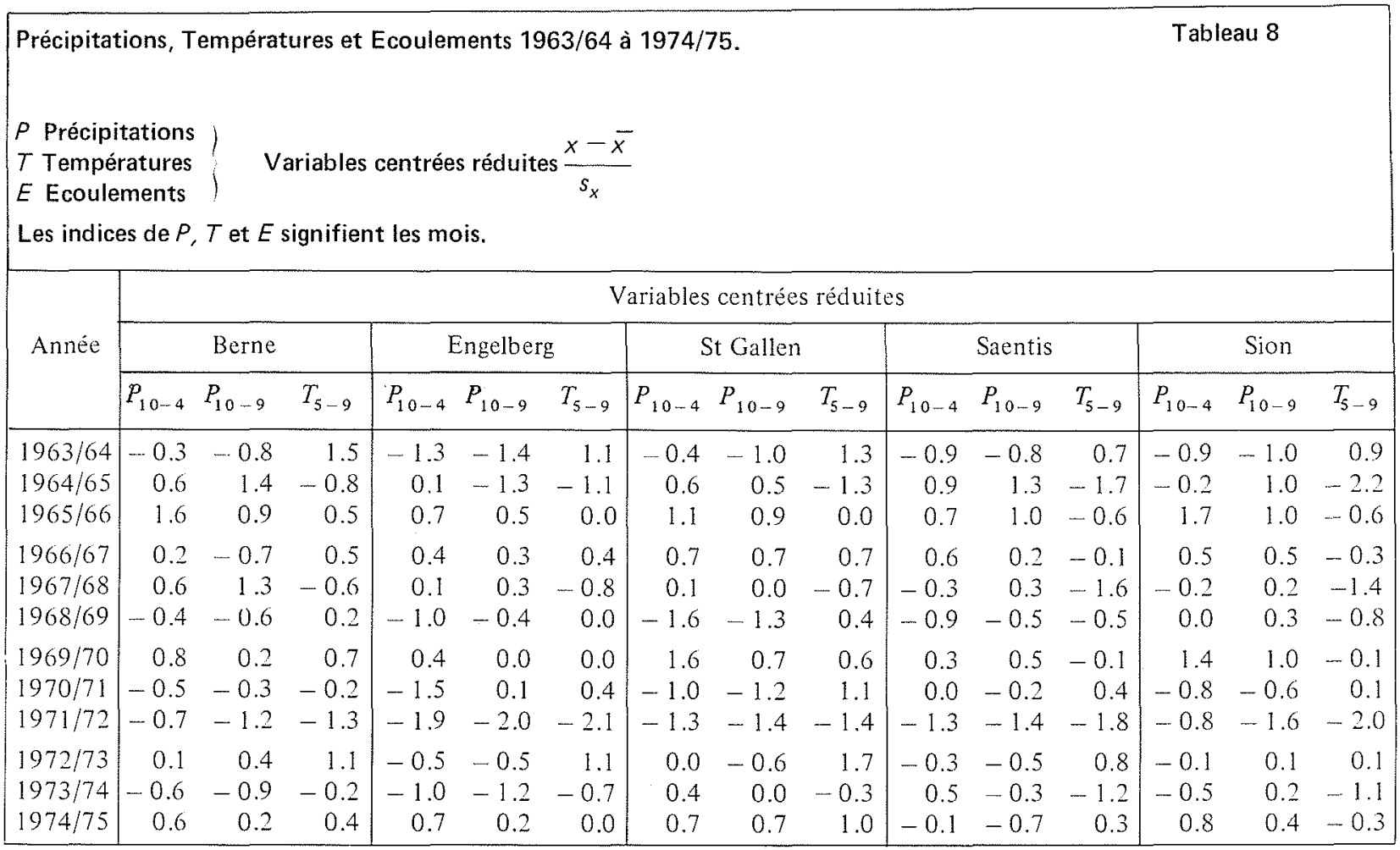

\begin{tabular}{|c|c|c|c|c|c|c|c|c|c|c|c|c|c|c|c|c|}
\hline \multirow{3}{*}{ Année } & \multicolumn{16}{|c|}{ Variables centrées réduites } \\
\hline & \multicolumn{3}{|c|}{ Chur } & \multicolumn{3}{|c|}{ Zermatt } & \multicolumn{3}{|c|}{ Locarno-Monti } & \multicolumn{3}{|c|}{ Davos-Platz } & \multirow{2}{*}{$\frac{\mathrm{Jj}\left({ }^{1}\right)}{\mathrm{T}_{\mathrm{s}-9}}$} & \multirow{2}{*}{$\frac{M B(2)}{E_{10-0}}$} & $R P\left({ }^{3}\right)$ & \multirow{2}{*}{$\frac{\operatorname{RR}\left({ }^{4}\right)}{E_{10-9}}$} \\
\hline & $\mathrm{P}_{10-4}$ & $P_{10-9}$ & $T_{5-9}$ & $P_{10-4}$ & $P_{10-9}$ & $T_{5-9}$ & $P_{10-9}$ & $P_{10-9}$ & $T_{5-9}$ & $P_{10-4}$ & $P_{10-9}$ & $\mathrm{~T}_{s-9}$ & & & $E_{10-9}$ & \\
\hline $1963 / 64$ & -0.4 & -1.5 & 1.8 & -0.5 & -1.0 & 1.6 & 1.2 & -0.1 & 1.2 & -0.9 & -1.7 & 0.3 & 1.3 & 0.3 & -1.7 & -1.3 \\
\hline $1964 / 65$ & 0.7 & 2.5 & -2.3 & -0.5 & 0.9 & -1.6 & -1.1 & 0.1 & -1.2 & 0.3 & 1.7 & -4.4 & -1.6 & -1.5 & -1.5 & 1.1 \\
\hline $1965 / 66$ & 0.6 & 1.6 & -0.4 & 1.5 & 0.3 & -1.2 & -0.6 & -0.6 & -0.2 & 0.5 & 1.2 & -1.3 & -0.2 & -1.2 & 0.8 & 1.6 \\
\hline $1966 / 67$ & 1.7 & 1.8 & 0.4 & 1.2 & 1.1 & -1.5 & 1.0 & 0.8 & -0.2 & 1.0 & 1.1 & -0.8 & 0.2 & -0.5 & 0.2 & 0.6 \\
\hline $1967 / 68$ & 1.1 & 1.1 & -0.6 & -0.7 & -0.3 & -2.1 & 0.6 & 0.7 & -1.6 & 0.9 & 0 . & -1.7 & -1.2 & -1.4 & -0.8 & 0.5 \\
\hline $1968 / 69$ & -0.9 & -0.9 & 0.4 & 0.7 & 1.0 & -1.5 & 0.1 & 0.2 & -0.1 & -0.9 & -0.5 & -0.1 & 0.1 & -1.0 & -0.5 & 0.0 \\
\hline $1969 / 70$ & 1.9 & 1.7 & 0.3 & 0.7 & 0.3 & -1.3 & -0.3 & -0.7 & 0.3 & 1.1 & 1.4 & -0.6 & -0.1 & -0.1 & 1.1 & 1.3 \\
\hline $1970 / 71$ & -0.7 & -1.0 & 0.6 & 0.0 & 0.2 & 0.0 & 0.2 & 0.0 & -0.2 & -0.5 & -1.1 & 0.1 & -0.2 & -0.2 & -1.1 & -1.1 \\
\hline $1971 / 72$ & -1.5 & -2.2 & -1.6 & 0.1 & 0.0 & n & 0.5 & -0.2 & -1.9 & -1.5 & -2.0 & -2.4 & -1.8 & -1.5 & -2.4 & -1.8 \\
\hline $1972 / 73$ & -0.2 & -0.5 & 1.1 & -1.3 & -0.2 & $n$ & -1.1 & -1.1 & 0.8 & -0.5 & -0.3 & 0.5 & 0.8 & -0.1 & -0.6 & -0.6 \\
\hline $1973 / 74$ & 0.9 & 0.9 & -0.6 & -0.6 & -0.6 & $\mathrm{n}$ & 0.3 & -0.4 & 0.1 & 1.1 & 1.6 & -1.6 & -1.3 & -1.1 & -1.5 & -0.4 \\
\hline $1974 / 75$ & 2.0 & 1.6 & 0.5 & 1.0 & 1.3 & $\mathrm{n}$ & 0.6 & 0.4 & 0.0 & 0.8 & 0.9 & -0.3 & -0.4 & -1.0 & 0.5 & 1.2 \\
\hline
\end{tabular}

(1) Jungfraujoch

(2) Blatten/Blatten-Naters
(3) Rhône/Porte du Scex

(4) Rhin/Rheinfelden stationnaires et en décrue pour toutes les séries d'années où l'on dispose d'observations. Sauf pour le glacier de Sarennes, l'année de bilan de 1964/65 montre une fin marquée d'une longue période de récession. L'augmentation du pourcentage des glaciers en crue pendant la DHI est certainement due à un léger rétablissement des glaciers. Mais il ne faut pas surestimer l'importance de cette évolution, du fait que pendant la longue période de récession, les glaciers ont perdu des aires englacées considérables avant tout dans leur zone d'ablation. Les conditions climatiques de la DHI qui ont été moins hostiles aux glaciers que celles des décennies précédentes, ont abaissé le niveau des lignes d'équilibre et augmenté la quote-part des zones d'accumulation par rapport aux zones d'ablation. Les conséquences de ce fait sont :

1 - L'ablation diminue par rapport à l'accumulation, ce qui facilite des bilans équilibrés ou mème positifs. Il dépend de l'évolution future du climat que la récession soit atténuée ou qu'un état plus ou moins stationnaire ou méme une période de crue générale intervienne. Les conditions climatiques moyennes pendant la DHI ne sont pas encore assez favorables aux glaciers pour engendrer une période de crue générale.

2 - Par rapport à la période de récession où l'écoulement a profité de la diminution de volume des glaciers. l'indice d'écoulement a diminué pendant la DHI. ce qui est une conséquence directe des pertes daires englacées dans les zones d'ablation. C'est une des conséquences importantes pour la production hydroélectrique des aménagements dans les bassins à haute glaciation. 


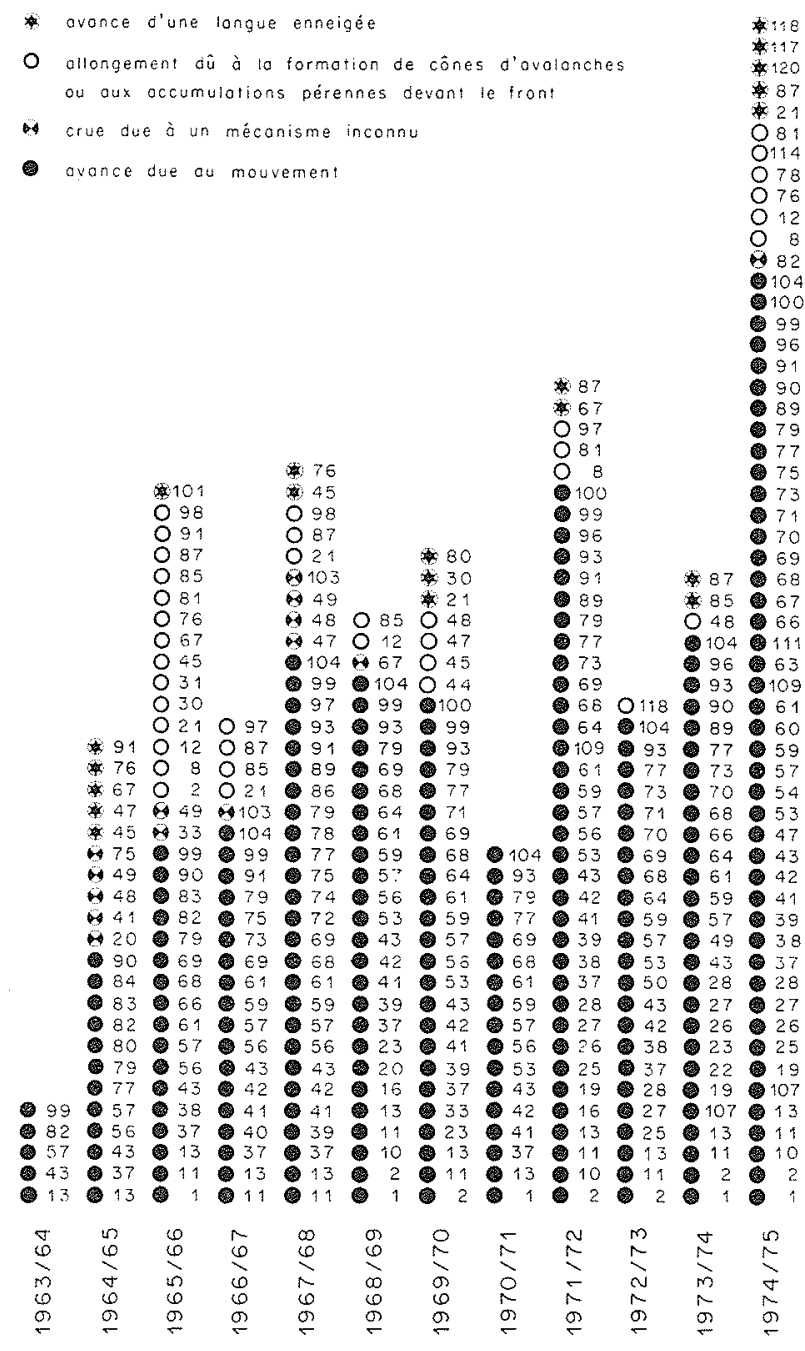

Les nombres inscrits dans le graphique sont les numéros d'identification GL des glaciers définis dans le tableau 1 .

6/ Les crues des années 1963/64 à 1974/75

Nombre de glaciers en crue et en décrue, en pourcent du nombre total des glaciers observés de $1891 / 92$ à $1974 / 75$.

Précipitations P10-9 et Écoulement $E_{10-9}$ du bassin versant de la Massa. Variables centrées réduites $\frac{x-\bar{x}}{S_{x}}$

Bilans annuels spécifiques en $\mathrm{gcm}^{-2}$ des glaciers de

Sa Sarennes

5 Aletsch

3 Gries

78 Limmern

90 Silvretta

$H$ Hintereis

$K$ Kesselwand

Sis Stubacher Sonnblickkees
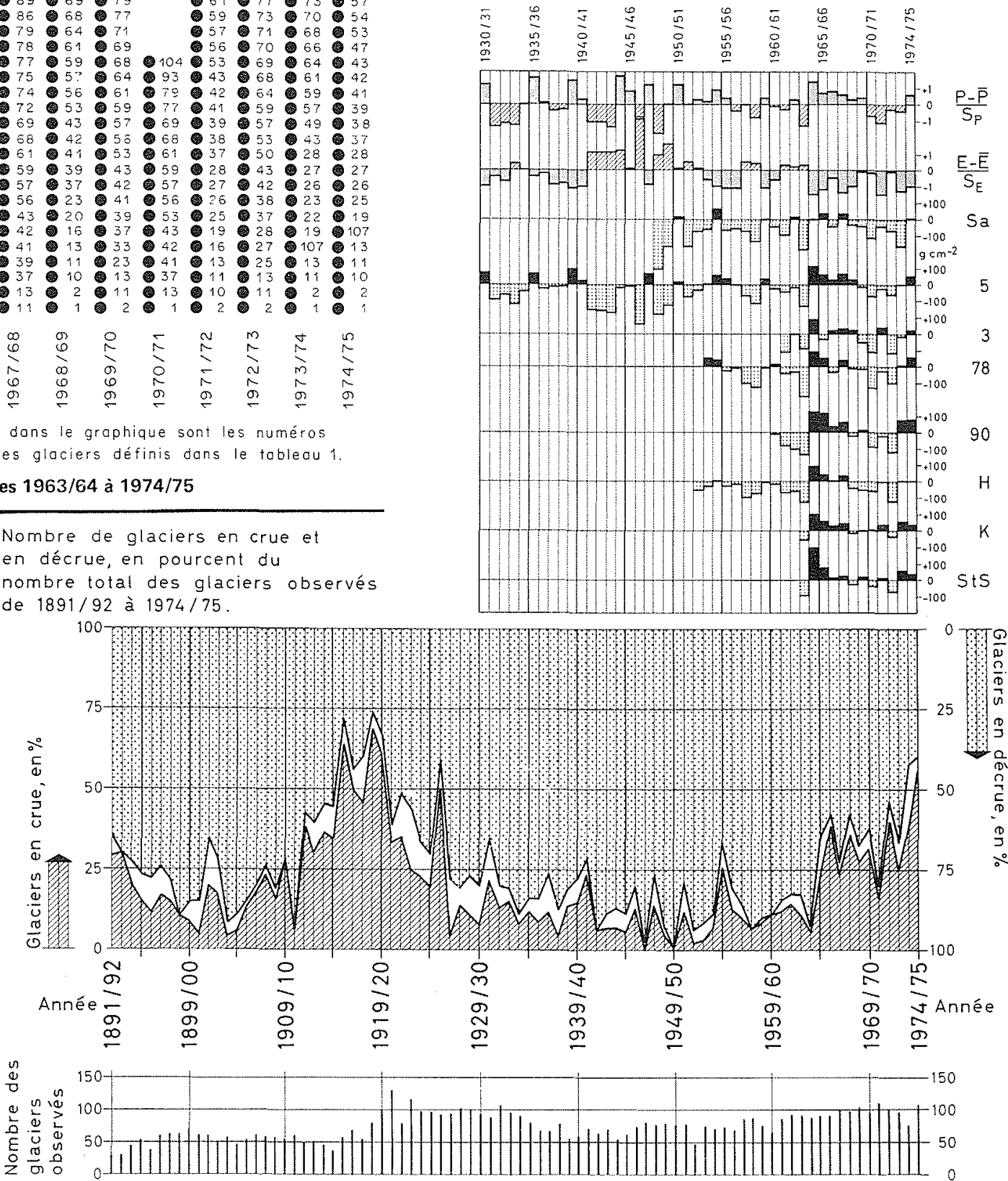

7/Précipitations et écoulements du bassin de la Massa, bilans annuels spécifiques de masse de différents glaciers et nombre de glaciers en crue et en décrue, en pourcent du nombre total des glaciers observés de 1891/92 à 1974/75. 


\section{Discussion}

Président : M.L. de CRECY

M. le Président remercie chaleureusement M. KASSER pour son exposé remarquablement documenté et illustré. Il ouvre ensuite la discussion.

Répondant à une question de M. OBLED, M. Le Président ne pense pas que l'utilisation et l'aménagement des glaciers pour le ski (zones de damage) apportent une perturbation notable du bilan annuel de l'ensemble car les surfaces intéressées sont relativement peu importantes.

Avez-vous noté, demande M. VIVIAN, une influence de l'altitude des langues terminales sur la variation glaciaire, indépendamment des problèmes d'orientation ou même de localisation régionale des variations?

J'ai commencé une étude sur ce point, répond $M$. KASSER mais je n'ai pas encore de résultats.

Il me parait intéressant de signaler que l'Institut de Géographie de l'Ecole Polytechnique de Zurich a établi, sous la direction de M. Fritz MULLER un inventaire complet des glaciers suisses sur la base d'une couverture aérienne réalisée en 1973.
Cet inventaire comprend maintenant 1828 glaciers ; mais on a pris en compte tous les petits névés pérennes à partir d'une surface d'un hectare et le plus grand nombre de ces 1828 glaciers sont des glaciers de petit appareil. Une publication sur ce sujet doit paraître à une date qui n'est pas encore précisée.

Grâce aux captages réalisés pour l'alimentation des usines hydro. électriques, dit M. BEZINGE, on a pu constater, en Suisse, que les moyennes des 15 dernières années des débits de fusion des petits glaciers avaient tendance à diminuer, en corrélation avec l'engraissement des glaciers. C'est une image immédiate de la "tendance" du climat au cours de cette période.

Il ne me reste plus qu'à remercier $M$. KASSER, conclut $M$. le Président. Nous éprouvons une grande admiration - et quelque envie - devant l'importance et l'ancienneté des travaux de la Commission des glaciers suisses. Nous avons encore beaucoup à faire, en France, avant d'arriver aux résultats remarquables que vous avez bien voulu nous présenter.

\begin{tabular}{|c|}
\hline $\begin{array}{l}\text { Abstract } \\
\text { Glacier variations in the Swiss Alps in } 1974 / 75 \text { and data obtained during } \\
\text { the International Hydrological Decade from } 1964 / 65 \text { to } 1973 / 74\end{array}$ \\
\hline 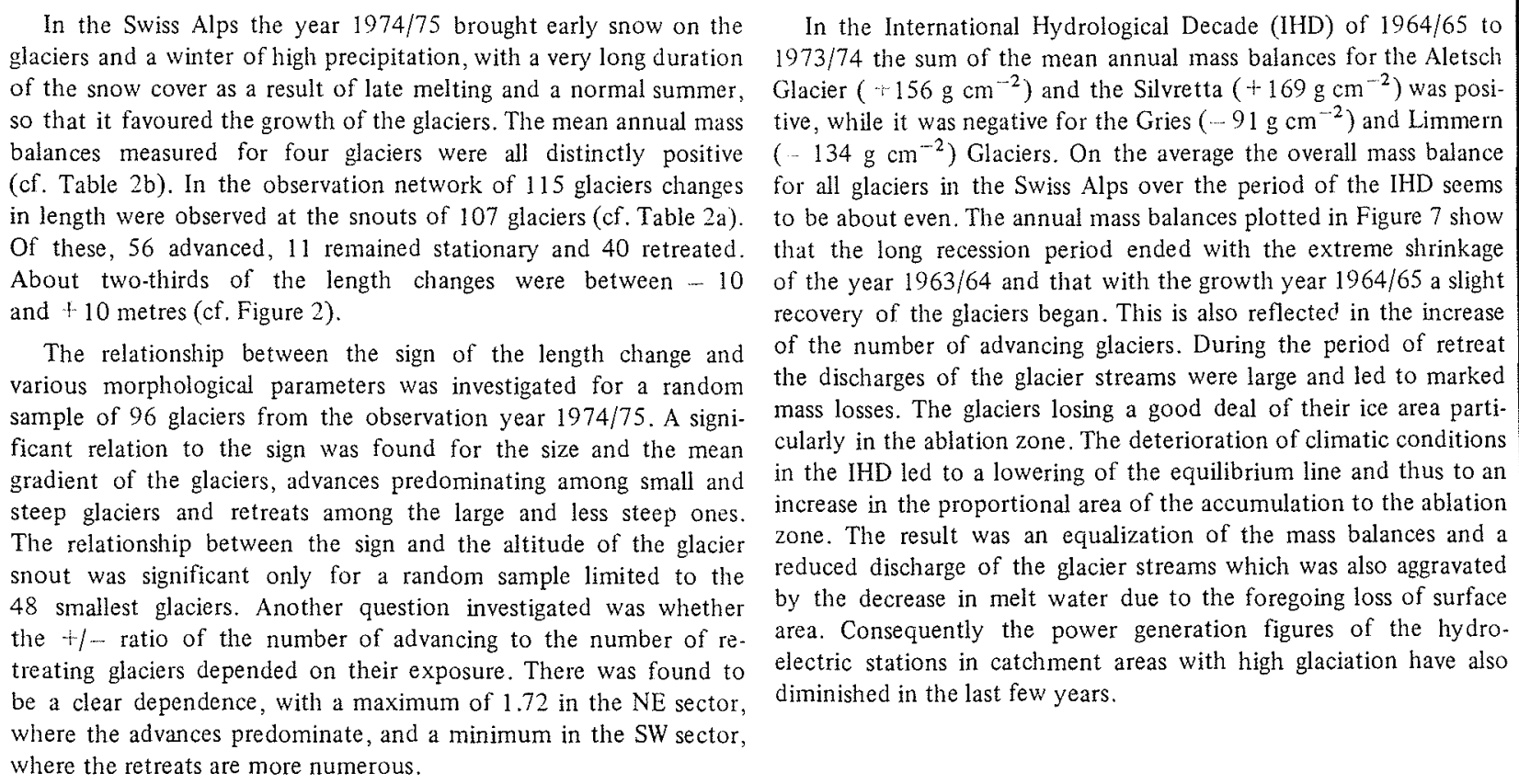 \\
\hline
\end{tabular}

